\title{
Le site du « Plateau de la Mayenne », un habitat rural des VIIe-IXe siècles aux portes d'Angers (Maine-et-Loire)
}

The site of the "Plateau de la Mayenne", a rural settlement of the 7th-9th centuries near Angers (Maine-et-Loire)

Der Fundplatz „Plateau de la Mayenne”, eine Siedlung des 7.-9. Jh. n. Chr. in der Nachbarschaft von Angers (Maine-et-Loire, France)

El sitio del "Plateau de la Mayenne", un habitat rural de los siglos VII-IX en los afueras de Angers (Maine-et-Loire)

Frédéric Guérin et Didier Pfost (avec la collaboration de)

\section{OpenEdition}

\section{Journals}

Édition électronique

URL : https://journals.openedition.org/rao/3106

DOI : $10.4000 /$ rao.3106

ISBN : 978-2-7535-5014-8

ISSN : 1775-3732

Éditeur

Presses universitaires de Rennes

Édition imprimée

Date de publication : 31 décembre 2015

Pagination : 363-384

ISBN : 978-2-7535-5012-4

ISSN : 0767-709X

Référence électronique

Frédéric Guérin et Didier Pfost (avec la collaboration de), «Le site du «Plateau de la Mayenne », un habitat rural des VIle-IXe siècles aux portes d'Angers (Maine-et-Loire) », Revue archéologique de l'Ouest [En ligne], 32 | 2015, mis en ligne le 28 avril 2018, consulté le 22 août 2022. URL : http:// journals.openedition.org/rao/3106 ; DOI : https://doi.org/10.4000/rao.3106 


\title{
Le site du " Plateau de la Mayenne », un habitat rural des VII ${ }^{\mathrm{e}}-\mathrm{IX}^{\mathrm{e}}$ siècles aux portes d'Angers (Maine-et-Loire)
}

\author{
The Site of the "Plateau de la Mayenne", a Rural Settlement of the $7^{\text {th }}-9^{\text {th }}$ Centuries \\ near Angers (Maine-et-Loire)
}

\author{
Frédéric GuÉrin ${ }^{\mathrm{a}}$, avec la collaboration de Didier PFOsT ${ }^{\mathrm{b}}$
}

\begin{abstract}
Résumé : Localisé au nord-ouest d'Angers, le site du "Plateau de la Mayenne » a été repéré en 2006 par X. Dubillot, lors d'un diagnostic archéologique. Reconnu sur plus de neuf hectares, l'établissement rural, daté des VII ${ }^{\mathrm{e}}$-IX ${ }^{\mathrm{e}}$ siècles, a fait l'objet d'une fouille en 2009 , fouille portant sur près de trois hectares. Dans ce périmètre, il a été possible d'étudier le cœur de l'occupation, matérialisé par un enclos, ainsi que ses abords immédiats, caractérisés par un système parcellaire orthogonal. Plutôt rares et mal conservées, les structures d'habitat se limitent à un bâtiment sur poteaux, un puits et, probablement, un four. Repérées en marge de l'habitat, quelques fosses et deux sépultures se rapportent également à l'installation. Celle-ci est abandonnée avant le $\mathrm{X}^{\mathrm{e}}$ siècle.
\end{abstract}

Abstract: Located northwest of Angers, the site of the "Plateau de la Mayenne" was discovered in 2006 by X. Dubillot, during an archaeological evaluation. Extending over an area of 9 hectares, 3 hectares of the rural settlement, dating to the $7^{\text {th }}-9^{\text {th }}$ centuries, was excavated in 2009. The excavation enabled the study of the heart of the occupation, materialized by an enclosure and an orthogonal field system. The poorly preserved features include a timber framed building, a well and probably an oven. Several pits and two graves are located on the periphery of the site. It is abandoned before the $10^{\text {th }}$ century.

Mots clés : haut Moyen Âge, habitat rural, enclos, fossés, bâtiment sur poteaux, puits, sépultures, mobilier.

Keywords: Early Middle Ages, rural settlement, enclosure, ditches, timber framed building, well, graves, assemblage.

\section{INTRODUCTION}

La réalisation d'un diagnostic archéologique ${ }^{2}$ préalable à l'aménagement d'une ZAC projetée au nord-ouest de l'agglomération angevine (fig. 1), à l'emplacement de l'ancien aérodrome d'Angers/Avrillé, a amené la découverte, au cours de l'été 2006, d'un important établissement rural du premier Moyen Âge : le site du "Plateau de la Mayenne ».

a Inrap Pays-de-la-Loire.

${ }^{\mathrm{b}}$ DAO, Inrap Pays-de-la-Loire.

1. Dubillot X., Angers/Avrillé (Maine-et-Loire), ZAC du Plateau de la Mayenne, tranche 1, 49007 et 49015, diagnostic archéologique, Rapport final d'opération, Inrap Grand-Ouest/SRA des Pays-de-la-Loire, Nantes, 2006.
Celui-ci, attesté sur une surface de neuf hectares, allait susciter, en 2009, la réalisation d'une fouille préventive ${ }^{2}$ portant sur près de trois hectares. Au sein de cette fenêtre (fig. 2), il a été possible d'étudier le cœur de l'installation, matérialisé par un enclos d'habitat, ainsi que ses abords immédiats, caractérisés par un réseau fossoyé orthogonal montrant des parcelles de tailles variables. Selon un mobilier céramique relativement abondant, l'installation paraît avoir été principalement occupée entre le VII ${ }^{e}$ et le $\mathrm{IX}^{\mathrm{e}}$ siècle. Outre des fos-

2. Guerin F., Angers (49007), Avrillé (49015), Maine-et-Loire, ZAC du Plateau de la Mayenne, tranche 1 : un établissement rural du haut Moyen Âge aux portes d'Angers, fouille archéologique, Rapport final d'opération, Inrap Grand-Ouest, Cesson-Sévigné, 2011. 


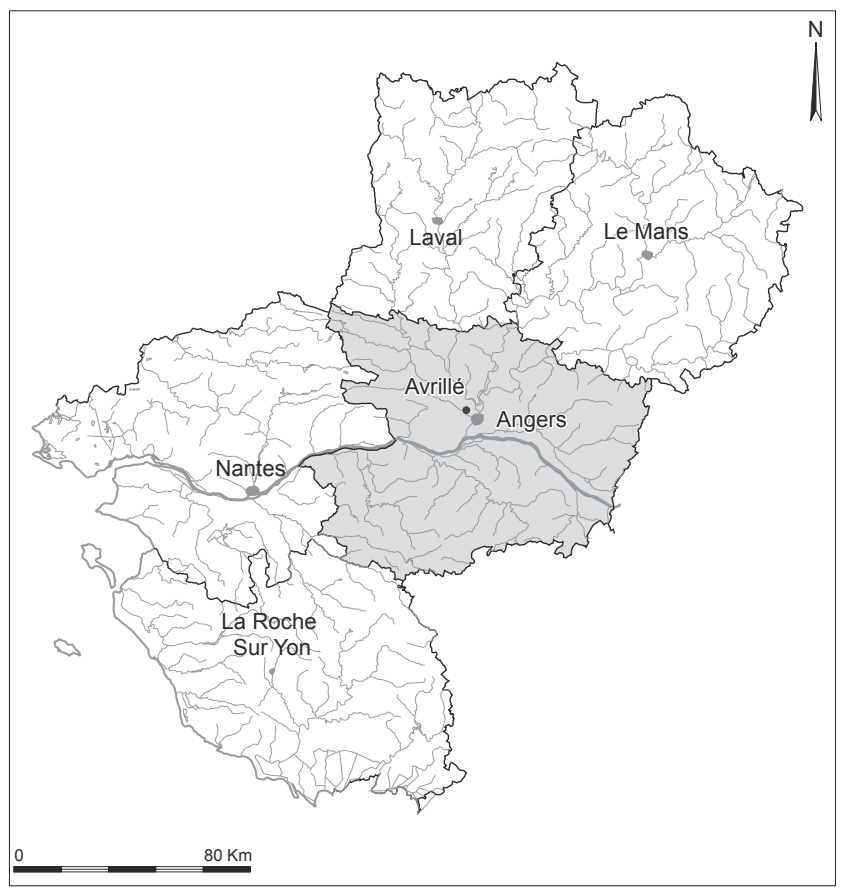

Figure 1 : Angers et Avrillé dans leur contexte régional. DAO D. Pfost, Inrap.

Figure 1: Anger and Arville in their regional context.
(O5-S3) attribués à l'Ordovicien supérieur et au Silurien ${ }^{3}$. Se rapportant au synclinorium d'Angers, ces formations apparaissent sous forme de plaquettes fragmentées de teinte orangé-gris ${ }^{4}$; ainsi structuré, le toit du rocher est recouvert par une argile limoneuse issue de l'altération du substrat. Si cette altérite est ponctuellement coiffée par des lambeaux d'alluvions anciennes relevant de hautes terrasses du Quaternaire, elle est avant tout scellée par un limon argileux brun-jaune : c'est dans ce dernier horizon qu'apparaissent les vestiges du haut Moyen Âge.

Aux fins de compléter cette présentation, on signalera la présence, sur les flancs nord et sud de l'anticlinal, de deux sources potentielles de matériaux dont des produits ou sousproduits sont attestés sur le site : les schistes d'Angers (O34), d'une part, et le minerai de fer de l'autre. Les premiers s'inscrivent à des distances comprises entre 50 et 300 mètres des vestiges médiévaux; de couleur bleu-noir à gris-bleuté, ils sont très fissiles; parmi eux, certains offrent, au sud, des qualités ardoisières. Second matériau proche du site altomédiéval, le minerai de fer se matérialise sous forme de minces couches inter-stratifiées, que ce soit au niveau du Grès armoricain situé au nord (Arenig : O2), ou bien au niveau du complexe des schistes et arkoses de Bains, établi au sud (Trémadoc et Arénig : O1-2); selon la formation considérée, une distance minimale de 300 à 600 mètres sépare le gisement des sources potentielles en minerai de fer.

\section{QUELQUES DONNÉES SUR L'ENVIRONNEMENT ARCHÉOLOGIQUE ET HISTORIQUE DU GISEMENT}

Avant la découverte de l'établissement rural du « Plateau de la Mayenne ", aucun témoignage archéologique n'était connu dans l'espace occupé par le gisement. En revanche, ses environs immédiats - volontairement limités aux confins nord-ouest de la commune d'Angers et au territoire communal d'Avrillé - ont livré différents vestiges s'insérant entre la préhistoire et le début de la période médiévale.

Issues de mentions anciennes, les traces relatives aux périodes préhistoriques - mal localisées - se limitent de surcroît à quelques artefacts : ainsi, deux bifaces de l'Acheuléen ancien sont-ils signalés dans les prairies inondables de la Mayenne, au nord-est de "La Perrière 5 ". Indicatifs du

3. Cavet P., Chauris L., Blaise J., Arnaud A., Gruet M., Lardeux H. et Brosse R., Carte géologique de la France à 1/50000, feuille d'Angers (454), éditions du BRGM, 1976, p. 11.

4. Deloze V., "Environnement naturel ", in Guerin F. (dir), Angers (49007), Avrillé (49015), Maine-et-Loire, ZAC du Plateau de la Mayenne, tranche 1: un établissement rural du haut Moyen Âge aux portes d'Angers, fouille archéologique, Rapport final d'opération, Inrap Grand-Ouest, Cesson-Sévigné, 2011, p. 39.

5. Dubillot X., op. cit., p. 5.

périmètre d'investigation repose sur des schistes et des grès 
Figure 2 : Plan des vestiges du haut Moyen Âge du site du «Plateau de la Mayenne». DAO D. Pfost, Inrap. Figure 2: Map of the Early Medieval site of "Plateau de la Mayenne".

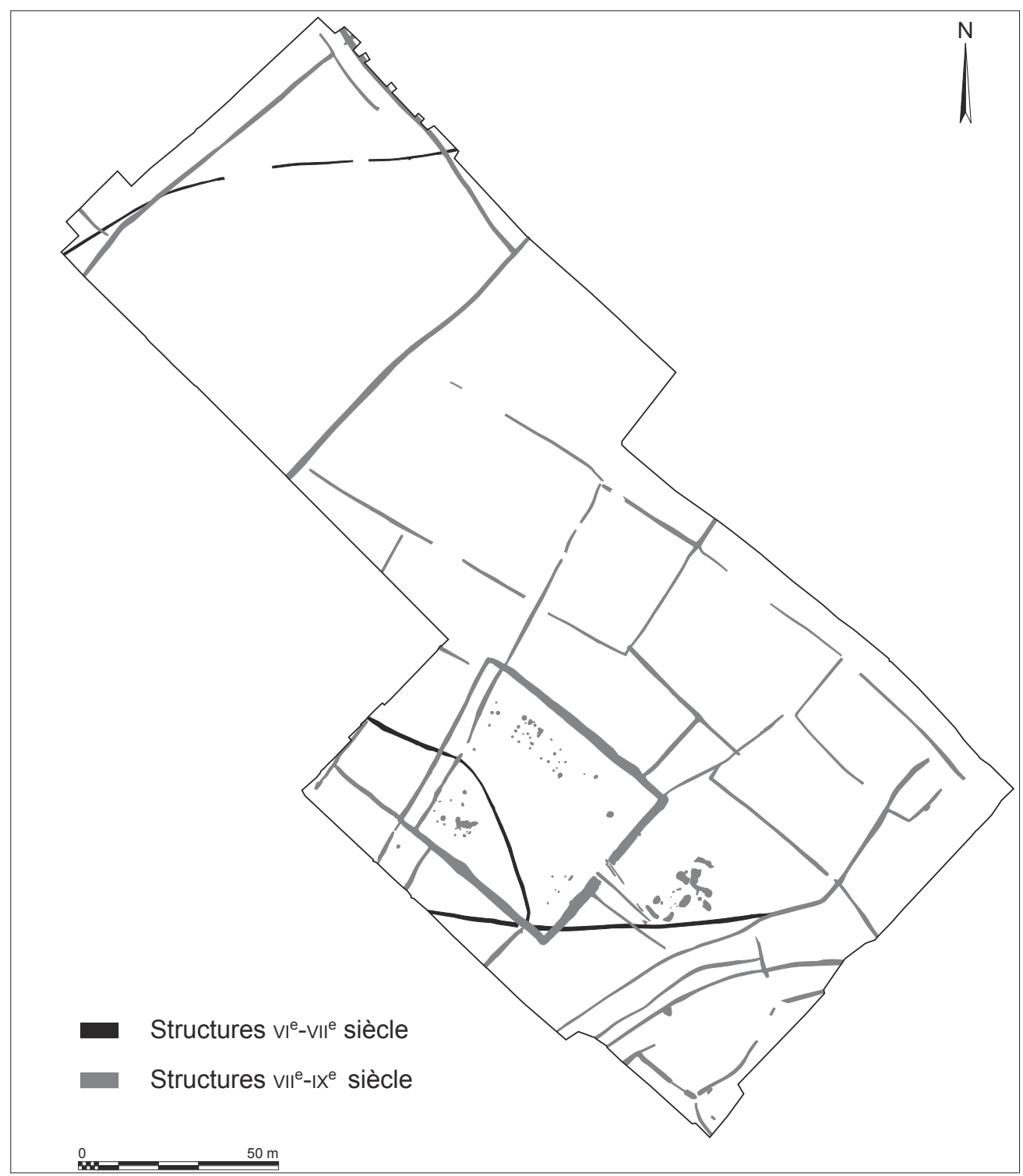

Néolithique, dix-sept outils en pierre polie proviennent quant à eux du "Bois du Roi ", en limite sud-ouest de l'ancien aérodrome $e^{6}$. Enfin, en "Reculée ", au nord d'Angers, le lit de la Maine a livré une hache polie ainsi que des bases d'andouillers de cerf'.

Grâce aux fouilles et diagnostics récents, la protohistoire est nettement mieux documentée; toutefois, selon la période considérée, les données sont plus ou moins nombreuses. Ainsi, âge du Bronze et premier âge du Fer ne sontils renseignés que par deux entités : "Le Tertre au Jau ${ }^{8}$ "

\section{Ibid., p. 5 .}

7. Desmazières O., «Inventaire des haches de pierre polie du département de Maine-et-Loire, et relevé des polissoirs ", Bulletin de la Société d'études scientifiques d'Angers, LV, 1925 p. 94.

8. Tourneur J., Angers/Avrillé (Maine-et-Loire), ZAC du Plateau de la Mayenne, Tranche 3, Rapport de diagnostic archéologique, Inrap GrandOuest/SRA des Pays-de-la-Loire, Nantes, 2007, p. 8, 9. et « Les Chalets ${ }^{9}$ ». Modestes ensembles archéologiques ne regroupant que quelques creusements, ces sites s'inscrivent au nord-ouest d'Angers (fig. 4).

Nettement plus marquée, l'empreinte du second âge du Fer s'exprime en effet par le biais de six gisements (fig. 4).

Établi au nord-ouest du bourg d'Avrillé, le site de " La Livonnière $^{10}$ » a livré les restes d'un système d'enclos à vocation agricole et domestique. Selon le mobilier céramique, il se rapporte à La Tène finale. Il en va de même de l'établis-

9. Guérin F., Angers (Maine-et-Loire), ZAC du Plateau des Capucins, lot $n^{\circ} 2$, rapport de diagnostic archéologique, Inrap Grand-Ouest/SRA des Pays-de-la-Loire, Nantes, 2008, p. 45.

10. Letho-Duclos Y., Avrillé (Maine-et-Loire), La Livonnière, Rapport de diagnostic archéologique, Inrap Grand-Ouest/SRA des Pays-de-laLoire, Nantes, 2003. 


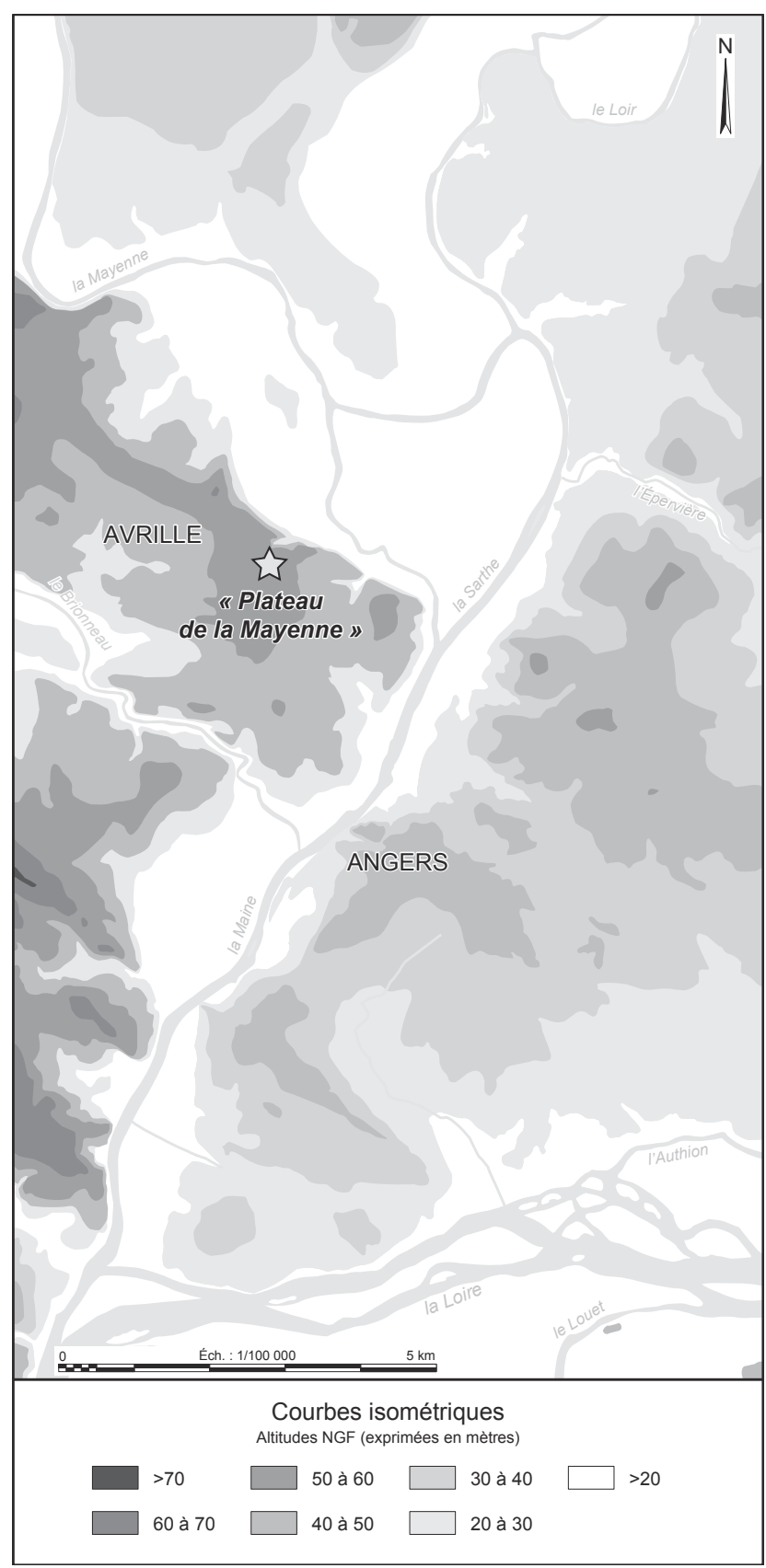

Figure 3 : Environnement topographique et hydrographique du site. Carte M. Pithon, Inrap; DAO D. Pfost, Inrap.

Figure 3: Topographic and hydrographic environment of the site.

sement rural de "Beauséjour ${ }^{11}$ ", où divers bâtiments sur poteaux prenaient place au sein d'une enceinte quadrangulaire; cette dernière, implantée sur le territoire d'Angers,

11. Pétorin N., Angers, Maine-et-Loire, Beauséjour, Établissement rural de La Tène finale, Fouille archéologique, Rapport final d'opération, Inrap Grand-Ouest, Cesson-Sévigné, 2010. était localisée à moins de cinq cents mètres au nord des vestiges du "Plateau de la Mayenne ».

Non loin de là, à "Tartifume $e^{12}$ ", c'est une portion d'enclos agricole de La Tène C2/D1 qui a été repérée. Plus au sud, au lieu-dit "La Fauconnerie ", un diagnostic a permis de révéler la présence d'un "fossé-limite » (?); daté lui aussi de La Tène C2/D1, il a par ailleurs fourni quelques tessons de La Tène moyenne, indices d'une occupation plus ancienne $e^{13}$.

Surplombant la vallée de la Mayenne, le site de " La Gatellière ${ }^{14}$ ", offre lui aussi des traces de La Tène moyenne : celles-ci se présentent sous la forme d'un petit enclos dont la fonction demeure problématique; reste que l'hypothèse d'un dispositif à finalité funéraire n'est pas à exclure ${ }^{15}$. Quoi qu'il en soit, à l'ouest de ce dernier, un système fossoyé comportant trois enclos est aménagé au cours de La Tène finale. Habitat agricole caractéristique de la fin de l'indépendance, il est lui-même environné au sud par un autre enclos : " La Gatellière 2 ». Ce dernier, associé éventuellement au précédent (?), se rapporte principalement à La Tène C2/D1 ${ }^{16}$.

Relativement nombreuses, les installations de La Tène finale révèlent la forte anthropisation qui caractérise alors les abords de l'oppidum des Andes vers la fin de l'indépendance gauloise. Peut-être liées à l'approvisionnement de la place forte (?), certaines de ces implantations paraissent abandonnées après la Conquête (Beauséjour); d'autres, au contraire, continuent d'être fréquentées (La Livonnière, La Gatellière).

Plus diffus, les témoignages antiques (fig. 4) n'en sont pas moins attestés aux abords du "Plateau de la Mayenne ». Ainsi, comme indiqué plus haut, le site de "La Gatellière » comporte-t-il également des structures de la période galloromaine; celles-ci se présentent notamment sous la forme de deux petits bâtiments sur fondations de pierres dont l'implantation reprend les orientations des fossés gaulois. Installés vers le début du Haut-Empire, les édifices prennent place au sein d'une occupation abandonnée définitivement vers la fin du $\mathrm{II}^{\mathrm{e}}$ siècle $^{17}$.

Voisin du précédent, le gisement de "La Gatellière 2 » offre lui aussi des traces gallo-romaines; en règle générale, celles-ci se manifestent sous forme de fossés parcellaires, fossés rapportés, largo sensu, à l'Antiquité. Également attes-

12. Dubillot X., op. cit., p. 12

13. Guerin F., Angers (Maine-et-Loire), ZAC du Plateau des Capucins, $\operatorname{lot} n^{\circ} 2$, Rapport de diagnostic archéologique, Inrap Grand-Ouest/SRA des Pays-de-la-Loire, Nantes, 2008, p. 46-51.

14. Hervé M.-L., Angers (Maine-et-Loire), La Gatellière, Rapport de fouille archéologique, Inrap Grand-Ouest/SRA des Pays-de-la-Loire, Nantes, 2004.

15. Ibid., p. 18, 19

16. Guerin F., Angers (Maine-et-Loire), ZAC du Plateau des Capucins, lot $n^{\circ} 2$, Rapport de diagnostic archéologique, Inrap Grand-Ouest/SRA des Pays-de-la-Loire, Nantes, 2008, p. 53-60.

17. Hervé M.-L., op. cit. 
Figure 4: (Voir planche couleur X) Environnement archéologique du site (commune d'Avrillé et confins nord-ouest d'Angers). Carte IGN 1522 O; DAO D. Pfost, Inrap.

Figure 4: (See colour plate X) Archaeological environment of the site (Avrillé and northwest of Angers).

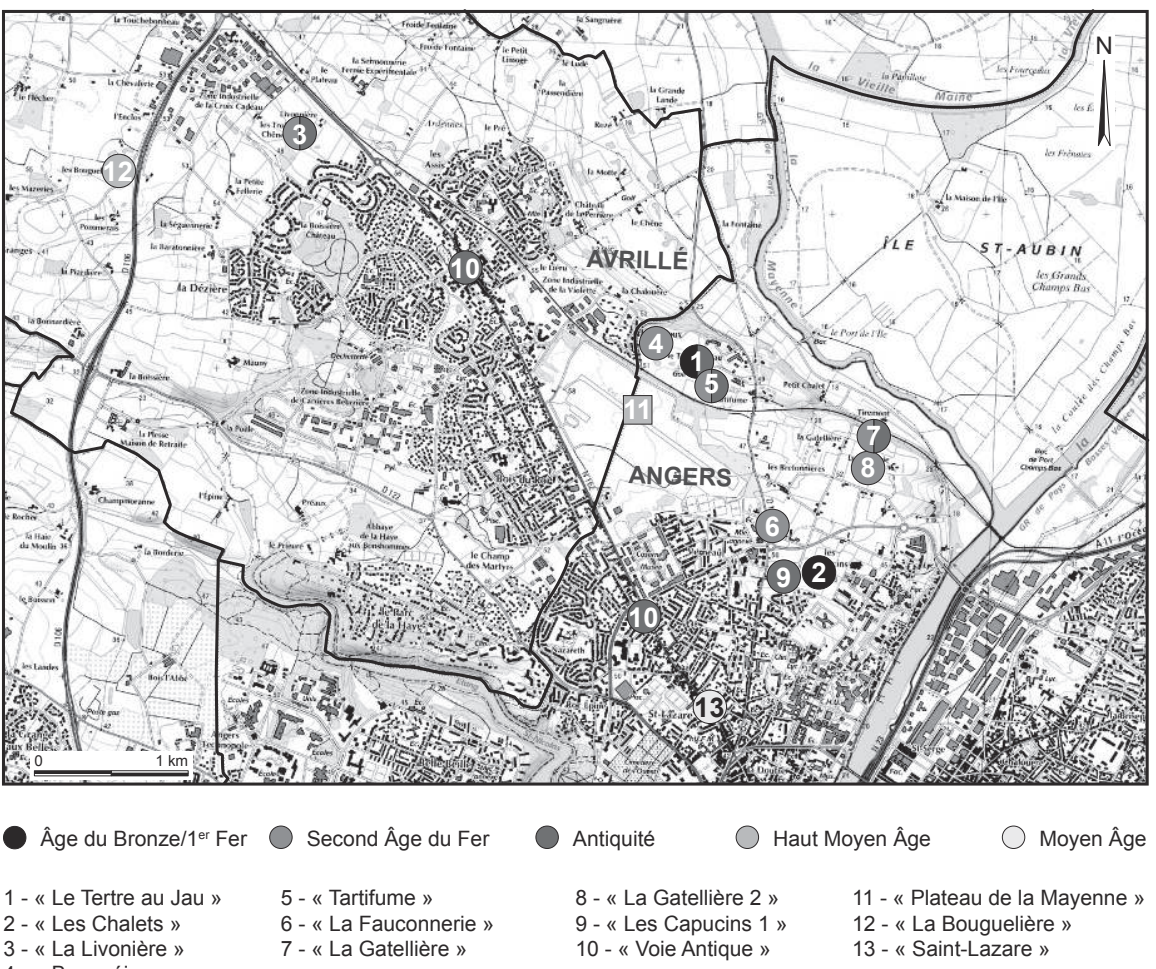

tés au niveau du "Plateau des Capucins ${ }^{18}$ ", divers creusement parcellaires sillonnent le périmètre; au vu des artefacts recueillis, une fréquentation au Haut-Empire peut être retenue. Signalés auprès de "Tartifume ", non loin du «Plateau de la Mayenne ", d'autres tronçons parcellaires structurent l'espace antique ${ }^{19}$; rattachés à la seconde moitié du $\mathrm{I}^{\mathrm{er}}$ siècle apr. J.-C., ils semblent s'organiser selon une dynamique héritée de La Tène finale.

Vecteur probablement important de l'organisation du paysage local, la voie qui reliait autrefois Juliomagus (Angers) à Condate (Rennes), passe à environ sept cents mètres au sudouest des vestiges du «Plateau de la Mayenne ${ }^{20}$ ». Traversant aussi bien Angers qu'Avrillé, l'axe viaire a été sondé sur la commune voisine du Plessis-Macé, à quelques kilomètres au nord-ouest du gisement ${ }^{21}$.

18. Guerin F., Angers (Maine-et-Loire), ZAC du Plateau des Capucins, tranche 1, Rapport de diagnostic archéologique, Inrap Grand-Ouest/SRA des Pays-de-la-Loire, Nantes, 2006, p. 4.; Guerin F., Angers (Maineet-Loire), ZAC du Plateau des Capucins, lot $n^{\circ} 2$, Rapport de diagnostic archéologique, Inrap Grand-Ouest/SRA des Pays-de-la-Loire, Nantes, 2008, p. 64-70.

19. Tourneur J., op. cit., p. 19.

20. Guerin F., Angers (49007), Avrillé (49015), Maine-et-Loire, ZAC du Plateau de la Mayenne, tranche 1 : un établissement rural du haut Moyen Age aux portes d'Angers, fouille archéologique, Rapport final d'opération, Inrap Grand-Ouest, Cesson-Sévigné, 2011, p. 66.

21. Fricot L., Avrillé, La Meignanne, La Membrolle-sur-Longuenée, Montreuil-Juigné, Le Plessis-Macé (Maine-et-Loire), «RD 775 (ex RN 162) - Aménagement en $2 X 2$ voies entre Avrillé et La Membrolle-sur-Longuenée,
Enfin, à l'instar de « La Gatellière », le gisement de « La Livonnière " (Avrillé), recelait lui aussi des traces d'occupation du Haut-Empire ${ }^{22}$. Établies à proximité de la voie romaine, certaines de ces structures semblent avoir été influencées par le dispositif viaire.

Au vu des données antiques issues des différents sites repérés aux abords nord-ouest de Juliomagus, il ressort l'image d'un secteur, qui à la période gallo-romaine, paraît principalement orienté vers des espaces accueillant des pratiques agro-pastorales. Dans un autre registre, on remarquera que si des témoignages du Haut-Empire sont signalés sur plusieurs gisements, en revanche, pour le Bas-Empire, il n'existe à ce jour aucun indice avéré.

Comparativement à La Tène finale et à l'époque antique, le haut Moyen Âge (fig. 4) se révèle faiblement documenté, puisqu'en dehors du site du "Plateau de la Mayenne", la période n'est illustrée que par une seule entité archéologique reconnue : "La Bouguelière ${ }^{23}$ ". Ne comprenant que quelques fosses et fossés, cette installation sise à Avrillé témoigne d'une modeste occupation domestique des VII ${ }^{\mathrm{e}}$ VIII $^{\mathrm{e}}$ siècles.

Enfin, quoi que plus tardives que le gisement évoqué ci-dessus, il importe néanmoins de signaler les sépultures

déviation de l'ETAS ", Rapport de diagnostic d'archéologie préventive, SAD de Maine-et-Loire/ SRA des Pays-de-la-Loire, Angers, 2007, p. 60-65.

22. Letho-Duclos Y., op. cit.

23. Fricot L., op. cit., p. 54-59. 
en coffrages de schiste retrouvées en 1991 sous la chapelle Saint-Lazare. Initialement attribuées au premier Moyen $\hat{A}_{\mathrm{ge}}{ }^{24}$, elles seraient en réalité liées au bâtiment $\mathrm{XI}^{\mathrm{e}}$ siècle dont la présence a pu être confirmée lors de la fouille de 2008 (renseignement oral F. Comte). Établie sur la commune d'Angers, en bordure de l'ancienne voie romaine, la première léproserie aurait donc été remplacée dans le courant du Xire siècle par un nouvel édifice.

Si les traces archéologiques du premier Moyen Âge font actuellement défaut au nord-ouest d'Angers, en revanche, quelques données relatives à l'histoire de la cité d'Angers, permettent de percevoir certains événements qui ont pu affecter, entre le milieu du v $v^{e}$ et la fin du Ix $x^{e}$ siècle, les abords de la ville. Déjà, dès la fin du III ${ }^{\mathrm{e}}$ ou du début du Iv ${ }^{\mathrm{e}}$ siècle, la construction de l'enceinte urbaine suggère une période de troubles.

Au milieu du v viècle, en 453, le concile d'Angers annonce l'excommunication "pour quiconque ouvrirait la ville à l'envahisseur barbare ${ }^{25}$ ", suggérant ainsi une ambiance générale conflictuelle. Un peu plus tard, vers 470-471, le comte Paul est tué en défendant Angers contre les pirates saxons ${ }^{26}$, ce qui sous-tend, là encore, une vie difficile en dehors des remparts.

A contrario, suite à "l'écroulement du royaume wisigoth de Toulouse en 507 la première moitié du $\mathrm{VI}^{\mathrm{e}}$ siècle, fut incontestablement une période favorable ${ }^{27}$ ". Faut-il y voir une relation de causes à effets avec le début de l'occupation du site du "Plateau de la Mayenne »?

Quoi qu'il en soit, on remarque que son abandon, au $\mathrm{IX}^{\mathrm{e}}$ siècle, coïncide peu ou prou avec le nouveau conflit avec la Bretagne. Après la défaite de Charles le Chauve face à Erispoë en 851, le traité d'Angers fixe, pour un temps, la frontière entre les deux provinces. C'est dans ce contexte que le comte, véritable chef de guerre, est installé sur le promontoire comme garde-frontière.

Dès lors, le territoire du "Plateau de la Mayenne " se trouvait une nouvelle fois au " premier rang de manœuvre de troupes, [contexte] peu propice à la sécurité. Les raids scandinaves qui suivirent - Angers y est confrontée en 854 et même occupée en 873 - eurent peut-être raison de l'installation de l'établissement agricole mis en évidence ${ }^{28}$ ».

24. Сомте F., "Le site, la chapelle et l'enclos des lépreux ", in SaintLazare: Histoire d'une Léproserie et d'un faubourg d'Angers, XII'-XVIT siècle, ARDA n ${ }^{\circ}$ 1, Angers, 1997, p. 8-15.

25. Tonnerre N.-Y., "L'Anjou de l'Antiquité tardive au très haut Moyen Âge ", Actes des XXII journées internationales d'Archéologie mérovingiennes tenues à Angers en 2001, à paraître.

26. Ibid.

27. Ibid.

28. Brodeur J., « Les abords nord-ouest d'Angers entre la fin de l'âge du Fer et le haut Moyen Âge ", in Guerin F. (dir.), Angers (49007), Avrillé (49015), Maine-et-Loire, ZAC du Plateau de la Mayenne, tranche $1:$ un établissement rural du haut Moyen Âge aux portes d'Angers, fouille archéo-

\section{Chronologie du site du « Plateau de la Mayenne »}

\section{Quelques traces antérieures à l'occupation du haut Moyen Âge}

En dehors de rares éclats de silex du Paléolithique supérieur retrouvés au sein de structures médiévales, les premières traces avérées de fréquentation du secteur se rapportent à l'Antiquité. Pour autant, relativement ténues, elles se limitent à une petite fosse ainsi qu'à des fragments résiduels de terres cuites architecturales parfois associés à quelques tessons, parmi lesquels deux formes du Bas-Empire ont été notées. Elles datent des $\mathrm{III}^{\mathrm{e}}-\mathrm{IV}^{\mathrm{e}}$ siècles. Quoi qu'il en soit, au regard des données collectées, ressort l'image d'un secteur faiblement marqué par la présence gallo-romaine; dans un autre registre, cette dernière ne paraît pas avoir exercé la moindre influence sur les vestiges du premier Moyen Âge.

\section{Phase $1\left(\mathrm{VI}^{\mathrm{e}}-\mathrm{VII}^{\mathrm{e}}\right.$ siècles) : des prémices à l'installation médiévale? (fig. 5)}

S'inscrivant dans un environnement où les traces antiques sont à peine perceptibles, l'occupation alto-médiévale ne se manifeste d'abord que sous l'aspect de trois fossés. Ces derniers, légèrement curvilignes, affectent des dimensions plutôt modestes : de fait, leur largeur s'inscrit entre 0,40 mètre et 1,20 mètre, tandis que leur profondeur ne dépasse pas les 0,50 mètre. Si, en l'absence de lien stratigraphique, il s'avère impossible de valider leur appartenance à un même dispositif, leur recoupement systématique par des creusements des VIII ${ }^{\mathrm{e}}$-IX $\mathrm{X}^{\mathrm{e}}$ siècles permet, en revanche, de supposer sinon leur synchronie, du moins leur rattachement à une même phase.

Cette première phase, caractérisée par les seuls fossés évoqués, est donc peu marquée dans le périmètre des fouilles. Sans finalité défensive, ces premiers témoignages médiévaux paraissent voués à la délimitation et au drainage des terrains environnants : en effet, régis par des orientations à dominante $\mathrm{O} / \mathrm{E}$, les creusements sont distinctement installés dans le sens de la pente. Pauvres en mobilier, ils ont toutefois livré quelques restes céramiques des $\mathrm{VI}^{\mathrm{e}}-\mathrm{VII}^{\mathrm{e}}$ siècles. Ces artefacts, recueillis principalement sur la frange sud-ouest du décapage, trahissent sans doute la présence - ou du moins la proximité - d'une implantation domestique.

Les vestiges, limités aux seuls fossés, témoignent probablement de l'existence d'espaces à vocation prioritairement agropastorale. Cette orientation fonctionnelle présumée pourrait s'être exercée au sein de "parcelles " matérialisées par des

logique, Rapport final d'opération, Inrap Grand-Ouest, Cesson-Sévigné, 2011, p. 40. 
Figure 5 : Plan phasé du site. DAO D. Pfost, Inrap.

Figure 5: Phased map of the site.

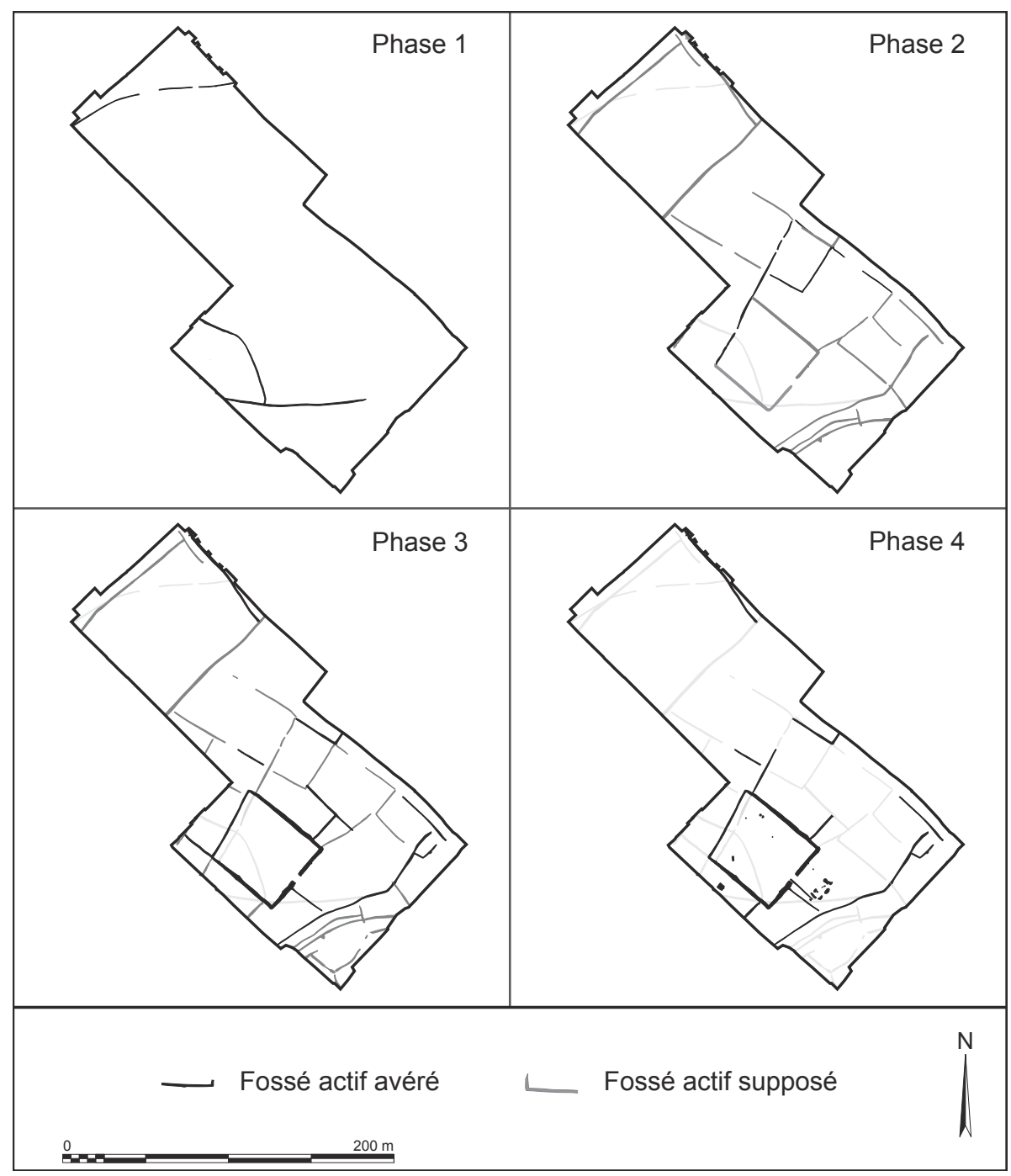

axes curvilinéaires qui, toute proportion gardée, évoquent quelque peu les fossés des enclos agricoles de « l'Écuyère 1 et $3 »$ ( $\mathrm{VII}^{\mathrm{e}}$-VIII ${ }^{\mathrm{e}}$ siècles), fouillés par N. Pétorin, dans la région des Mauges, au sud-ouest de l'Anjou ${ }^{29}$. En définitive, les premières traces alto-médiévales du "Plateau de la Mayenne " vont être recoupées par les éléments constitutifs de la phase suivante, phase caractérisée par une autre dynamique spatiale

\section{Phase 2 (VII ${ }^{\mathrm{e}}-\mathrm{VIII}^{\mathrm{e}}$ siècles?) : mise en place des éléments " orthogonaux » (fig. 5)}

Bien distincte de la précédente, la deuxième phase d'occupation médiévale se matérialise par la mise en place d'un dispositif fossoyé orthogonal : celui-ci va structurer l'implan-

29. Pétorin N., Mercier-Doucet F. et Pean E., Cholet (Maine-etLoire), ZAC L'Écuyère, diagnostic archéologique, Rapport final d'opération, Inrap Grand-Ouest/SRA des Pays-de-la-Loire, Nantes, 2004. p. 32. tation durant toute la durée de l'occupation. Sous-tendue par les orientations NNE/SSO d'une part, et ONO/ESE de l'autre, l'organisation spatiale du gisement s'articule peutêtre déjà autour de l'enclos central.

Il est vrai qu'initialement, la façade septentrionale de l'enceinte s'étire alors en dehors des limites de l'enclos, filant ainsi en direction du nord-est; là, avec d'autres segments, le creusement participe d'un schéma orthogonal strict dont l'ancienneté est attestée par des relations de chronologie relative.

Loin d'amener une augmentation des artefacts médiévaux, la mise en place, au cours des $\mathrm{VII}^{\mathrm{e}}$-VIII ${ }^{\mathrm{e}}$ siècles, de la trame parcellaire orthogonale, s'accompagne au contraire d'une diminution du nombre des restes céramiques (NR : 12 ; NMI : 4$)^{30}$ puisque ces derniers figurent en effet en plus

30. Coffineau E., "La céramique du haut Moyen Âge ", Guerin F. (dir.), Angers (49007), Avrillé (49015), Maine-et-Loire, ZAC du Plateau de la Mayenne, tranche 1 : un établissement rural du haut Moyen Âge aux portes 
petit nombre que ceux de la phase précédente (NR : 50 ; NMI : 7) ${ }^{31}$. À l'aune de ces données, le périmètre apparaît donc toujours principalement orienté vers des pratiques agro-pastorales.

En fin de compte, la configuration orthogonale qui sous-tend dès lors le canevas fossoyé du gisement, n'a rien d'exceptionnel pour le haut Moyen Âge. C'est ainsi qu'en contexte régional, le site des "Landes de Beaulieu ", dont l'acmé se situe entre le $\mathrm{VI}^{\mathrm{e}}$ et le viII ${ }^{\mathrm{e}}$ siècle ${ }^{32}$, a livré la partie d'une installation rurale, dont les fossés étaient, pour la plupart, régis par des orientations perpendiculaires. Un peu plus éloigné, mais toujours situé sur l'ancien territoire de l'Anjou, le site de "Vauvert » à Château-Gontier ${ }^{33}$, a pour sa part permis d'observer un ensemble complexe d'enclos rectangulaires ou carrés se structurant progressivement, entre la fin du viII ${ }^{\mathrm{e}}$ et la période carolingienne, autour d'un noyau parcellaire « central ». Quant au gisement alto-médiéval du "Teilleul ", à l'est de la Bretagne ${ }^{34}$, il a offert l'opportunité de percevoir un ensemble de parcelles de plan carré ou rectangulaire définissant un système fossoyé parfaitement organisé.

\section{Phase 3 (VIII ${ }^{\mathrm{e}}-\mathrm{IX}^{\mathrm{e}}$ siècle ?) : renforcement de l'occupation (fig. 5)}

S'affirmant de manière très nette au $\mathrm{VIII}^{\mathrm{e}}$-IX $\mathrm{X}^{\mathrm{e}}$ siècles - notamment au travers un mobilier céramique conséquent - l'occupation du "Plateau de la Mayenne " n'en reprend pas moins les cadres définis lors de la phase précédente. Quelques modifications sont cependant apportées. Au nombre de celles-ci, figure au premier chef, l'agrandissement de l'enclos dont la façade septentrionale est repoussée de cinq mètres vers le nord (fig. 6), tandis que ses flancs est, ouest et sud, sont élargis, provoquant ainsi la disparition quasi-totale de l'état initial de l'enceinte dont seul un lambeau a pu être observé au niveau du fossé sud-ouest. Avec ces travaux, l'enclos montre dès lors sa morphologie définitive.

C'est également à partir de cette phase qu'est attestée, auprès de l'angle nord-est de l'enceinte, la présence d'un bâtiment sur poteaux. À l'extérieur de l'enclos, non loin de l'entrée, c'est un groupe de différentes fosses qui atteste, au sud-est, du renforcement de l'occupation du site. Toujours

d'Angers, fouille archéologique, Rapport final d'opération, Inrap GrandOuest, Cesson-Sévigné, 2011, p. 174.

31. Ibid., p. 174.

32. GuÉRin F., Beaulieu-sur-Layon (Maine-et-Loire), Les Landes de Beaulieu, DFS de fouille, Afan/SRA des Pays-de-la-Loire, Nantes, 1999, p. 98.

33. Valais A., Arthuis R., Nauleau J.-F. et Morera-Vinçotte I., « Les enclos du haut Moyen Âge de Vauvert à Château-Gontier (Mayenne) ", Revue archéologique de l'Ouest, n' 26, Rennes, 2009, p. 205-227.

34. Catteddu I. (dir.), Les habitats carolingiens de Montours et La Chapelle-Saint-Aubert (Ille-et-Vilaine), DAF no 89, Paris, 2001, p. 36-39. à l'extérieur de la zone enclose, divers axes parcellaires sont créés, ou sans doute plutôt recreusés, tandis que d'autres, témoignent, au travers leur mobilier céramique, de leur appartenance à cette étape de l'histoire du gisement.

Avec 1469 fragments ${ }^{35}$ représentant un NMI de 124, cette phase est sans conteste la plus riche en matière de restes céramiques. Ceux-ci, constitués de formes à finalité domestique, proviennent essentiellement des fossés de l'enclos ainsi que du groupe de fosses installées hors de l'enceinte. Ailleurs, les éléments céramiques restent généralement fragmentaires et épars, hormis au niveau d'un fossé installé à une centaine de mètres au nord de l'enclos.

\section{Phase 4 (IX $-\mathrm{X}^{\mathrm{e}}$ siècle?) : abandon du site (fig. 5)}

Dans le courant du IX ${ }^{e}$ siècle s'amorce une diminution des traces de fréquentation, diminution qui aboutit - sans doute avant le début du $\mathrm{x}^{\mathrm{e}}$ - à l'abandon définitif du gisement. Certes, avec 785 fragments $^{36}$ représentant un NMI de 60, les lots céramiques restent conséquents. Malgré cela, ils n'en révèlent pas moins une nette diminution du corpus vis-à-vis de la phase précédente. En définitive, si l'on excepte un possible aménagement prenant la forme d'un empierrement interprété comme un accès secondaire (?) ménagé sur le flanc sud-ouest de l'enclos, c'est avant tout l'image d'une occupation " figée » qui ressort : en effet, à l'absence apparente de reprises d'éléments anciens ou d'installation de nouvelles structures, s'ajoute le comblement des différents fossés et creusements, qui, pour certains d'entre eux - les plus proches de l'habitat - vont dès lors faire office de dépotoirs.

Bien entendu, les causes qui ont présidé à l'abandon du site - qu'elles soient propres à l'installation elle-même ou liées à des facteurs externes - nous restent inconnues. Néanmoins, on signalera que le secteur où s'inscrit l'installation est, vers le milieu du $\mathrm{Ix}^{\mathrm{e}}$ siècle, le théâtre de troubles générés tant par le conflit opposant le royaume carolingien à la Bretagne que par les incursions scandinaves.

Quoi qu'il en soit, en dehors de ces événements qui affectent notamment la région angevine, E. Peytremann a pu constater l'existence d'un léger pic d'abandons au $\mathrm{IX}^{\mathrm{e}}$ siècle dans les Pays-de-la-Loire ${ }^{37}$, alors qu'à l'échelle du Nord de la France, l'acmé des désertions s'inscrit plutôt, quant à lui au $\mathrm{X}^{\mathrm{e}}$ siècle $^{38}$. Phénomène complexe, ce mou-

35. Coffineau E., op. cit., p. 174.

36. Ibid., p. 174.

37. Peytremann E., Archéologie de l'habitat rural dans le nord de la France du IV au XIT siècle, tome XIII des Mémoires publiés par l'Association française d'archéologie mérovingienne, Saint-Germain-en-Laye, 2003, vol. 1, fig. 101, p. 245.

38. Ibid., fig. 121, p. 256. 
vement de disparition des installations rurales du haut Moyen Âge - également noté dans une publication dirigée par A. Valais ${ }^{39}$ - relève sans doute d'une dynamique allant au-delà du simple " résultat d'une réorganisation des campagnes ${ }^{40} »$.

\section{LES PRINCIPAUX ÉLÉMENTS CONSTITUTIFS DE L'INSTALLATION AUX VII ${ }^{\mathrm{E}}-\mathrm{IX}^{\mathrm{E}}$ SIĖCLES}

\section{Un dispositif majeur de l'établissement : l'enclos d'habitat (fig. 6 et 7)}

Repéré au sud-ouest du décapage, l'enclos d'habitat constitue l'élément majeur de l'implantation. De plan trapézoïdal, il renferme une surface de 2200 mètres carrés. Ayant fonctionné ouvert, le fossé d'enclos affiche une profondeur maximale de 1,05 mètre; sa largeur oscille entre 0,75 mètre et 2,90 mètres. Modeste au nord-ouest, le creusement s'ouvre davantage au niveau des côtés sud-ouest et nord-est du dispositif, tandis qu'au sud-est, il présente sa plus grande largeur. Loin d'être fortuit, cet aspect répond

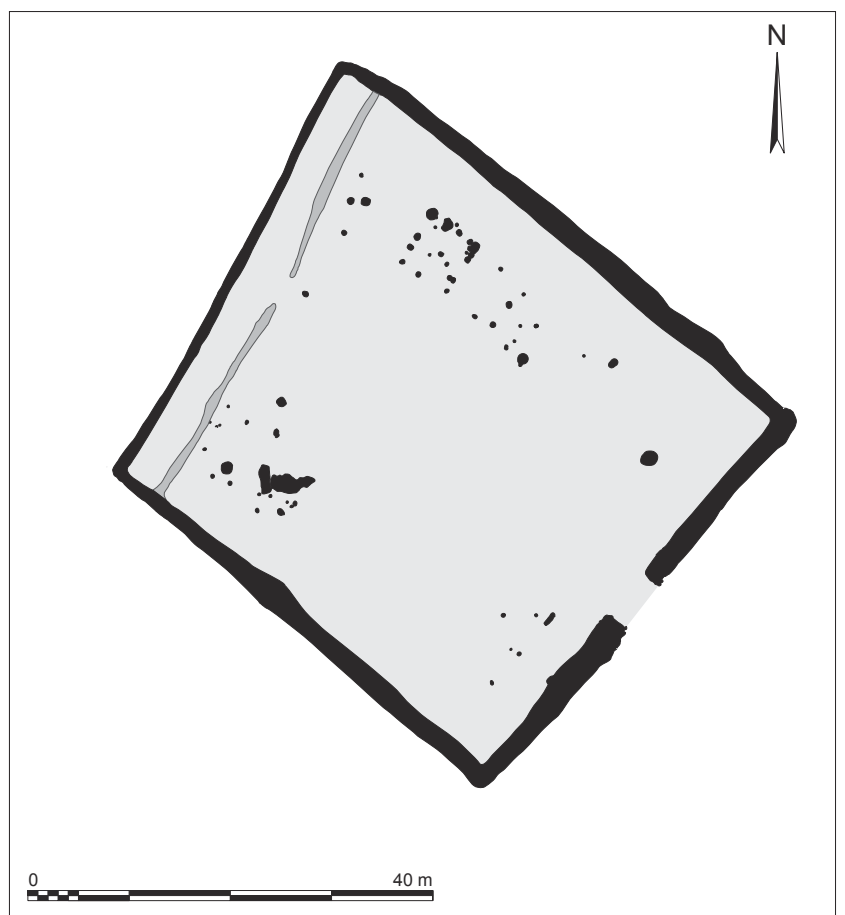

Figure 6 : Plan de l'enclos d'habitat. DAO D. Pfost, Inrap. Figure 6: Plan of the settlement enclosure.

39. GuÉRIN F., "L'organisation spatiale des établissements ruraux du Moyen Âge ", in Valais A. (dir.), L'habitat rural au Moyen Âge dans le NordOuest de la France, tome $1:$ Les synthèses, Rennes, PUR, coll. «Archéologie et Culture", Rennes, 2012, p. 81.

40. Peytremann E., op. cit., p. 357.

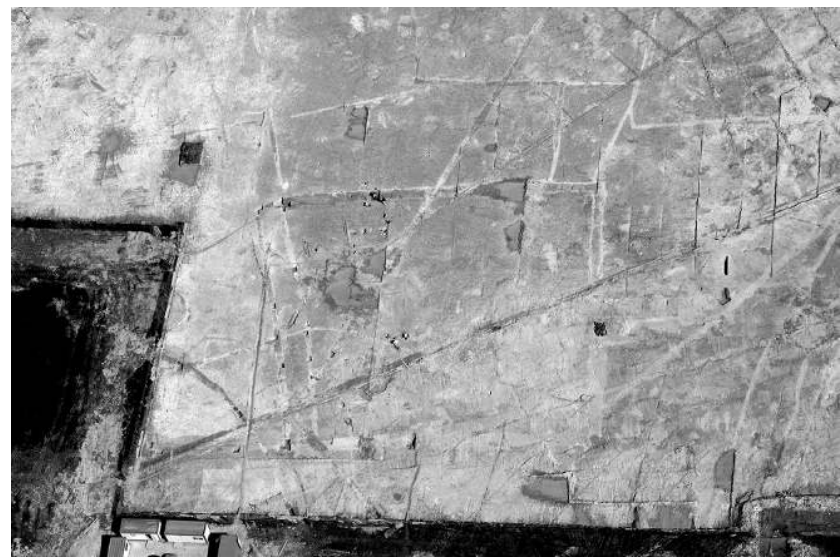

Figure 7 : (Voir planche couleur XI) Vue aérienne de l'enclos d'habitat. Cliché H. Paitier, Inrap.

Figure 7: (See colour plate XI) Aerial view of the settlement enclosure.

peut-être à une volonté ostentatoire (?), en lien avec la présence de l'accès à l'enclos; matérialisé par l'interruption du fossé, l'accès affiche une largeur de cinq mètres.

Compte tenu de l'absence de structure sur le bord interne du creusement, il est possible que le fossé ait été pourvu d'un talus (?); reste que la présence d'un tel aménagement demeure hypothétique. En définitive, il apparaît que la surface enclose ne renfermait qu' un petit nombre de structures : parmi les plus significatives, il a été noté la présence d'un bâtiment sur poteaux au nord, de quelques fosses à l'ouest et d'un foyer au sud. Quant au grand espace vide localisé au centre du dispositif, peut-être marque-t-il l'emplacement d'une cour (?); toutefois, là encore, un tel postulat reste à considérer avec prudence (fig. 6 et 7).

\section{Un bâtiment sur poteaux (fig. 8)}

Établi à l'intérieur de l'espace fossoyé, non loin de l'angle nord de l'enclos, le bâtiment prend place à environ cinq mètres du fossé nord-est du dispositif. Assemblage de poteaux plantés, l'édifice se développe selon l'orientation $\mathrm{ONO} / \mathrm{ESE}$ d'une part et selon l'axe NNE/SSO, d'autre part. Offrant un plan carré de 5,50 mètres de côté, il occupe une surface d'un peu plus de trente mètres carrés. Bien que peu probable, une extension pourrait s'être développée en direction du sud-est, portant ainsi l'ensemble à environ cinquante-six mètres carrés.

Parmi les vingt-deux creusements rapportés à l'édifice, quatorze semblent participer à l'ossature des parois. Si, pour la plupart d'entre eux, les avant-trous montrent des diamètres compris entre 0,50 mètre et 0,75 mètre, les plus importants, constitutifs de la paroi nord-est, affichent quant à eux jusqu'à 1,30 mètre d'ouverture pour 0,70 mètre de 


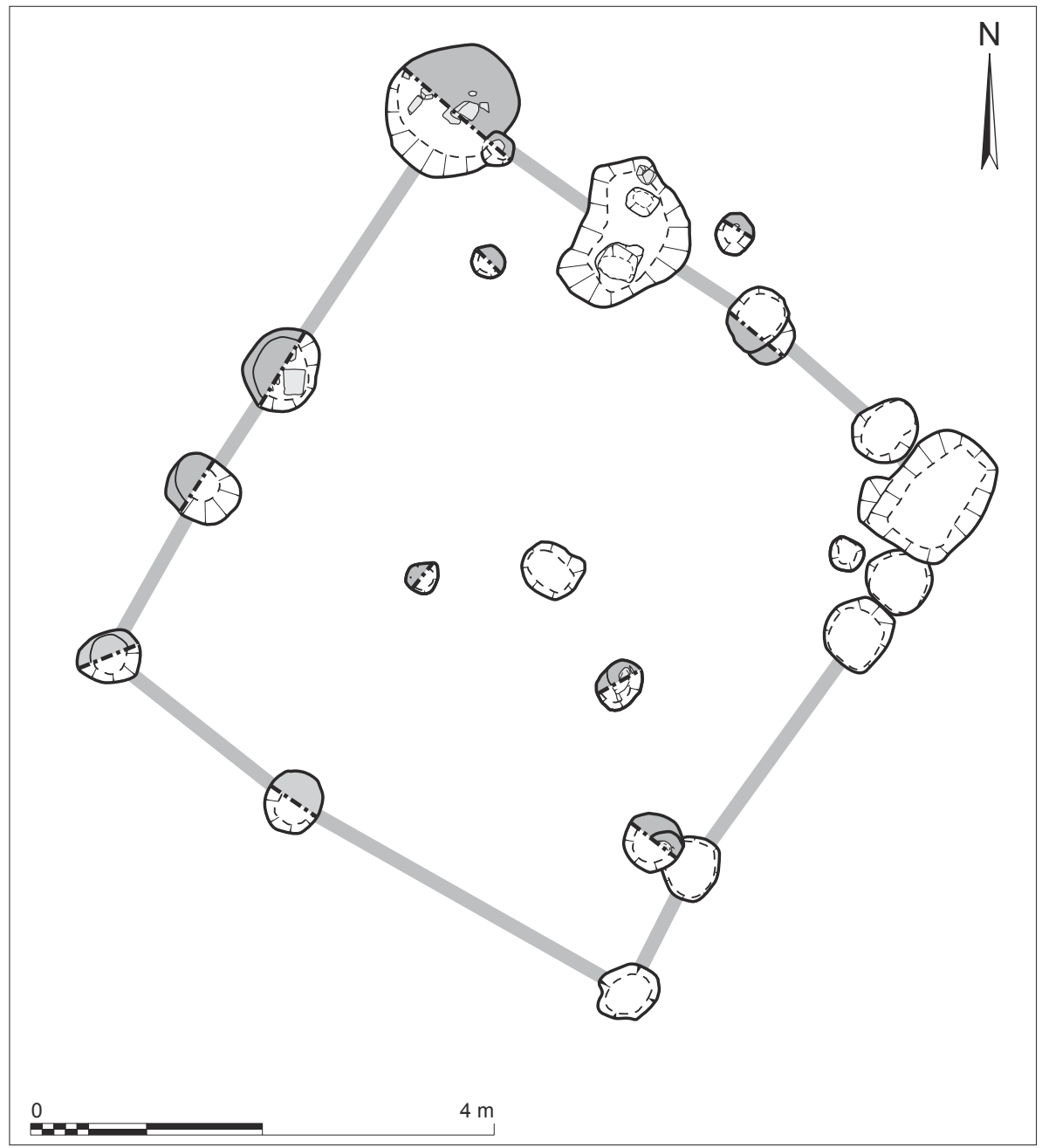

Figure 8 : Plan du bâtiment. DAO D. Pfost, Inrap.

Figure 8: Plan of the building.

profondeur. Établis dans l'emprise de la construction, les autres creusements pourraient témoigner de la présence d'aménagements internes ou faire office de support de faîtière : cette dernière fonction est en tout cas envisageable s'agissant du trou de poteau repéré au centre même du bâtiment. Quoi qu'il en soit, en dehors des blocs de calage, certains creusements ont également livré des négatifs de poteau; ceux-ci suggèrent l'implantation de pieux aux diamètres compris entre 24 et $30 \mathrm{~cm}$. Des relations de chronologie relative entre quelques trous de poteau attestent de réparations ou de réaménagements ponctuels.

L'édifice n'ayant fourni ni lambeau de sol, ni structure spécifique, il s'avère délicat de déterminer sa fonction; toutefois, des rejets caractéristiques (os d'animaux, tessons de céramique et de verre, fragment de meule) recueillis à proximité, tendent à rattacher le bâtiment à la sphère domestique (fig. 8).

\section{Les autres structures établies dans l'enclos}

Outre le bâtiment et divers trous de poteau, l'enclos renferme également quelques fosses ainsi qu'un foyer. Pour la plupart, les premières s'inscrivent auprès de l'angle ouest du dispositif fossoyé; deux d'entre elles, peu profondes $(0,25 \mathrm{~m})$ et de plan irrégulier, ont été interprétées comme fosses d'extraction; toutefois, la présence de mobilier en leur sein suggère une utilisation ultérieure comme structures de rejet. Sise non loin des deux précédentes, une troisième fosse, plus modeste (1,20 m de diamètre), semble, quant à elle, avoir été utilisée d'emblée comme structure de rejet : au vu de la forte fraction charbonneuse présentée par son remplissage, il s'agit probablement d'une "fosse-cendrier ".

Seule structure de cuisson médiévale repérée dans l'aire d'habitat, un foyer rectangulaire de 1,42 mètre de long pour 0,45 mètre de large (fig. 9) vient s'inscrire au sud de l'enclos, à proximité de l'entrée. Implanté parallèlement et à 3,50 mètres du côté sud-est de l'enceinte, le foyer montre un impact thermique nettement plus marqué dans sa moitié 


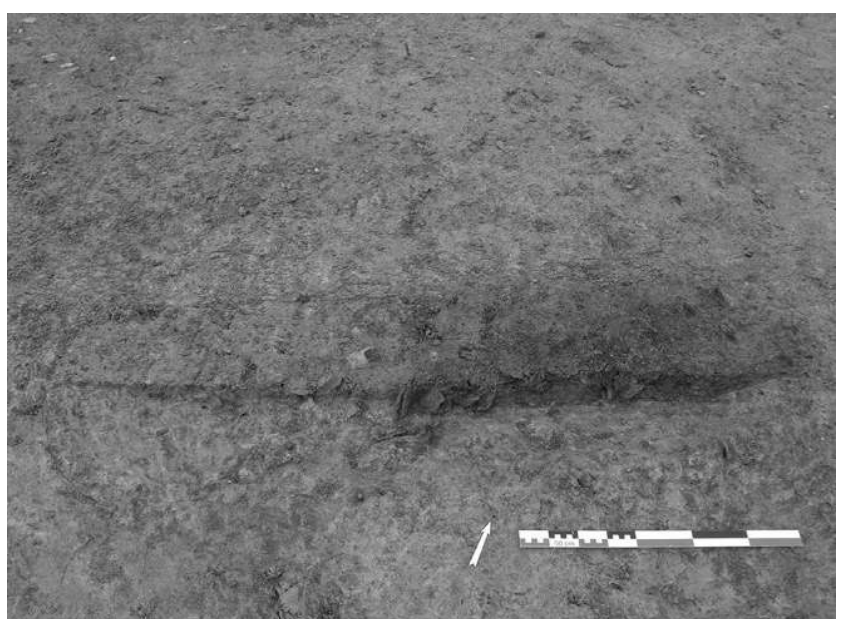

Figure 9 : (Voir planche couleur XII) Vue du foyer établi à l'intérieur de l'enclos. Cliché C. Luzet, Inrap.

Figure 9: (See colour plate XII) View of the hearth located in the enclosure.

nord. Quoi qu'il en soit, environné par une grande quantité de particules de charbon de bois associées à différents restes scoriacés, ce poste de cuisson témoigne peut-être de la présence, au sein de l'enclos, d'un petit artisanat métallurgique (forge?; fig. 9).

\section{Des éléments liés à l'habitat sis hors de l'enclos}

S'inscrivant sur le pourtour de l'enceinte fossoyée, non loin de ses limites sud-ouest et sud-est, différents vestiges indicatifs de pratiques artisanales, domestiques et agricoles viennent compléter l'installation. Ainsi, en va-t-il d'un foyer établi à environ six mètres au sud de l'angle ouest de l'aire d'habitat. Pourvu d'un diamètre de 0,70 mètre, il est composé d'un radier de tegulae que surmonte une sole d'argile rubéfiée (fig. 10). Tout comme le poste de cuisson précédemment évoqué, il s'inscrit à proximité d'un secteur caractérisé par d'abondants épandages charbonneux. Au vu de ses dimensions et de son mode de construction, il n'est pas sans rappeler un élément des $\mathrm{IX}^{\mathrm{e}}-\mathrm{X}^{\mathrm{e}}$ siècles - interprété comme four - retrouvé à l'extérieur d'un des bâtiments du site des " Murailles » à Distré, près de Saumur ${ }^{41}$ (fig. 10).

Sis à proximité immédiate de ce foyer, un puits (fig. 11 et 12) s'insère également à environ six mètres du côté sud-ouest de l'enclos. D'une profondeur supérieure à 4,70 mètres, le conduit est installé dans une fosse d'environ 3,20 mètres de côté; ce creusement, sorte d'avant-trou sans doute destiné à faciliter la tâche des constructeurs (?), a été remblayé par différents matériaux (limon argileux, plaquettes de schiste) apportés au fur et à mesure de la construction

41. VAlais A., Distré (Maine-et-Loire), Les Murailles, DFS de sauvetage urgent, Afan/SRA des Pays-de-la-Loire, Nantes, 1997, tome 1, p. 51.

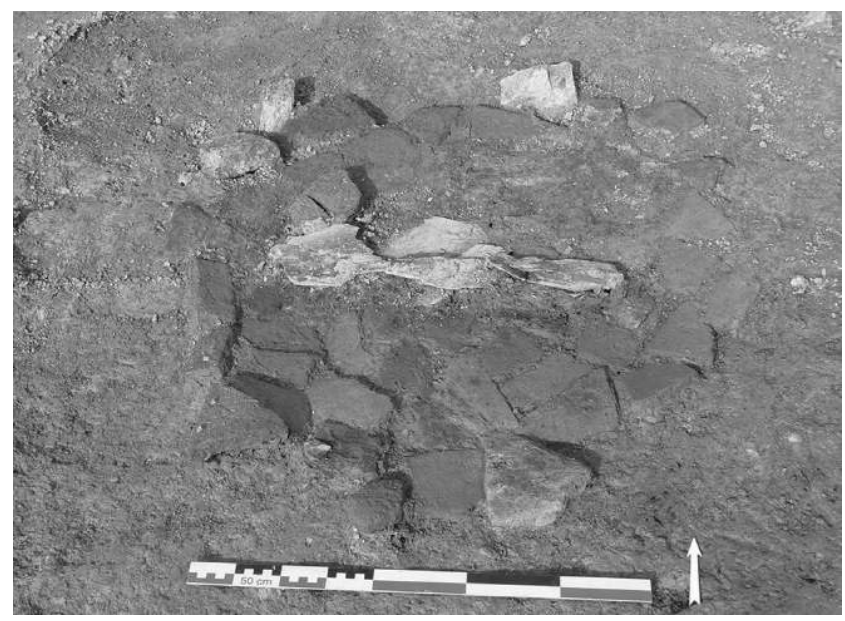

Figure 10 : (Voir planche couleur XII) Vue du foyer établi à l'extérieur de l'enclos, auprès de l'angle ouest. Cliché P. Matignon, Inrap. Figure 10: (See colour plate XII) View of the hearth located outside of the enclosure near to its west corner.

du cuvelage. Assemblage de blocs de quartz et de schiste liés entre eux par de l'argile, ce dernier affiche un diamètre de 1,05 mètre. Comblé par des niveaux argileux riches en pollens, le conduit a par ailleurs livré des tessons des ViII e$\mathrm{X}^{\mathrm{e}}$ siècles. Ultérieurement perturbé par une fosse vouée à la récupération d'une partie du cuvelage, le puits témoigne du caractère avant tout domestique du périmètre dans lequel il s'inscrit (fig. 11 et 12).

Prenant place au sud-est de l'enclos, à environ treize mètres de l'entrée, quelques trous de poteau et une structure de combustion côtoient une dizaine de creusements (fig. 13) au nombre desquels figurent plusieurs fosses évoquant des silos. Dans cette perspective, la présence d'une petite zone d'ensilage ne serait donc pas à exclure. Quoi qu'il en soit, circonscrit au sud-ouest et au nord-est par deux creusements oblongs (4,60 m à 5,10 $\mathrm{m}$ de long; $0,80 \mathrm{~m}$ à 1,20 $\mathrm{m}$ de large), ce périmètre comporte six fosses de plan ovale ou sub-trapézoïdal, d'une largeur comprise entre 1,50 mètre et 2,20 mètres, pour une longueur s'échelonnant de 2,10 mètres à 3,80 mètres; pourvues d'un fond plat associé à l'amorce de parois évasées, ces fosses étaient conservées sur une profondeur s'étageant de 0,35 mètre à 0,80 mètre.

Établis à peu de distance de l'enclos d'habitat, ces différents creusements ont assurément servi - dans un second temps de fosses de rejets; pour autant, si ces vestiges font penser à des silos, on ne peut cependant pas écarter d'autres alternatives parmi lesquelles figurent notamment celles d'excavations générées par l'extraction de matériau argileux, ou de dispositifs en lien avec des pratiques artisanales (?). Reste qu'indépendamment de l'hypothèse retenue, le secteur semble témoigner d'une zone d'activité « spécifique » (fig. 13). 


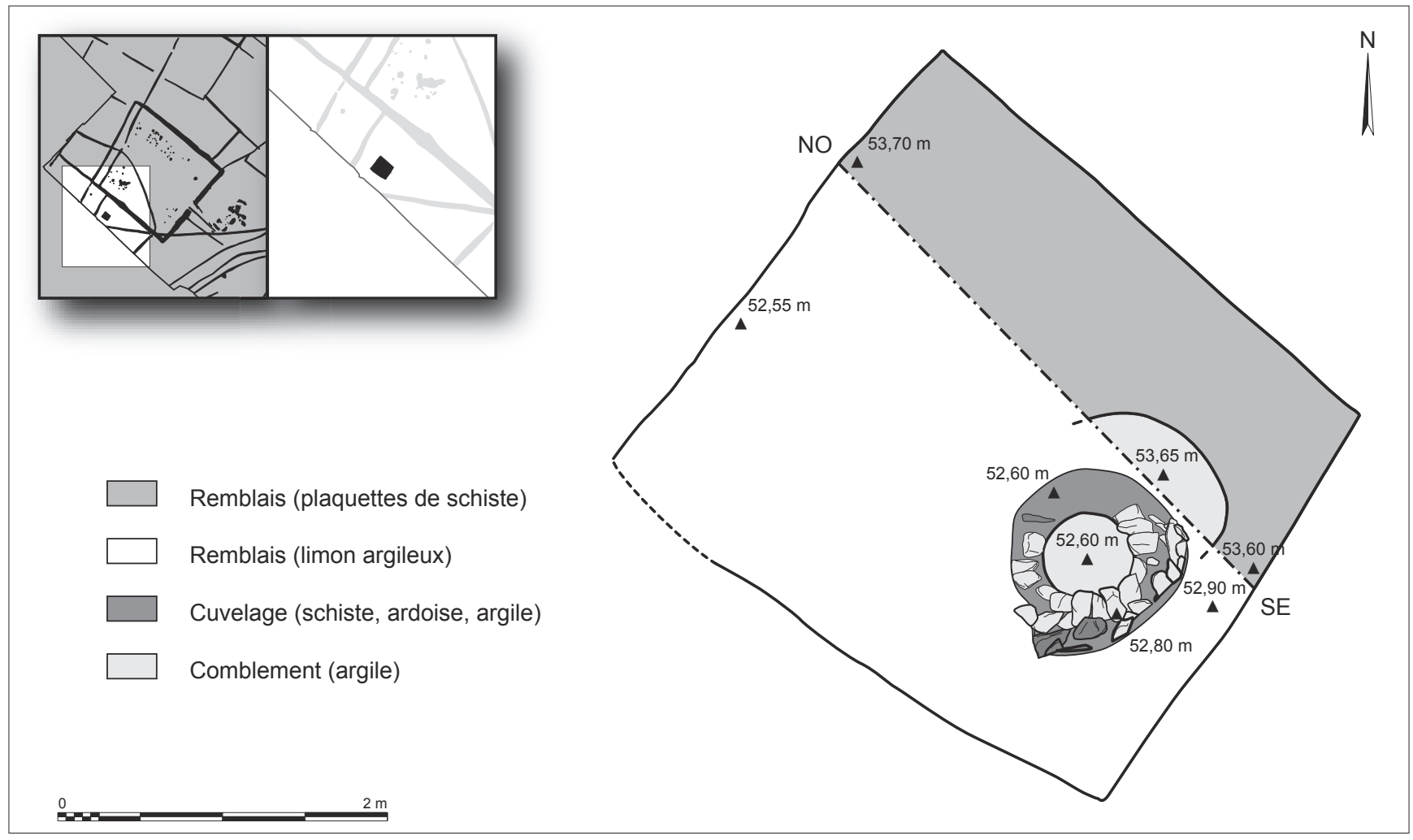

Figure 11 : Plan de la partie supérieure du puits. DAO D. Pfost, Inrap.

Figure 11: Plan of the upper section of the well.

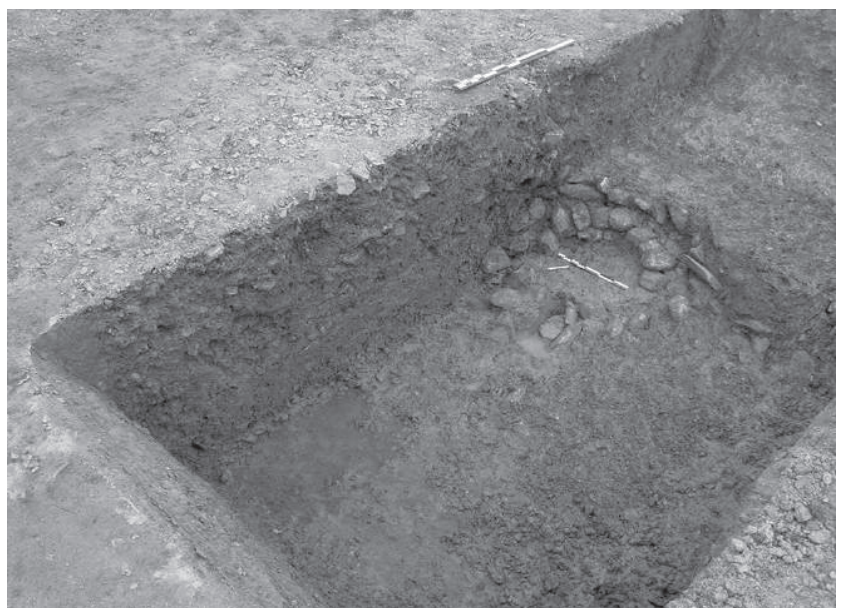

Figure 12 : Vue de la partie supérieure du puits. Cliché S. Brousse, Inrap.

Figure 12: View of the upper section of the well.

\section{Des sépultures isolées?}

Repérées à l'extrémité méridionale du décapage, à environ cinquante-cinq mètres au sud-est de l'enclos (fig. 14), deux sépultures prennent place en bordure de fossés. Distantes de six mètres l'une de l'autre, ces tombes en coffrage de schiste ardoisier montrent des orientations et des états de conservation variables; quoi qu'il en soit, vides d'ossements, elles n'ont, en outre, pas livré de mobilier (fig. 14).

Installée le long d'un fossé, la plus complète de ces structures funéraires (fig. 15) se développe selon l'orientation $\mathrm{NE} / \mathrm{SO}$. De plan rectangulaire, elle affiche une longueur de 1,50 mètre, pour une largeur de 0,58 mètre. Conservée sur une profondeur de 0,17 mètre, la fosse sépulcrale était délimitée par des panneaux constitués d'un assemblage irrégulier de plaques d'ardoise disparates. Outre les côtés, le fond de la fosse a également été aménagé au moyen de trois dalles de schiste ardoisier. Offrant des épaisseurs moyennes de $2 \mathrm{~cm}$, certaines d'entre elles comportaient des perforations (fig. 15).

Sise au sud de la précédente, la seconde sépulture s'est avérée nettement moins bien conservée : de fait, un chablis a profondément perturbé la tombe ne laissant subsister qu'une dalle de fond et deux plaques latérales. Reste qu'en dépit de leur ténuité, ces vestiges se sont révélés suffisamment pertinents pour déterminer l'orientation NO/SE du coffrage.

Trahissant peut-être l'existence d'un petit "noyau funéraire " situé aux marges de l'installation, les deux coffres de schiste ardoisier témoignent en tout cas d'une pratique récurrente au sein des établissements alto-médiévaux. Ainsi, caractéristiques de cette architecture faisant appel au schiste, 


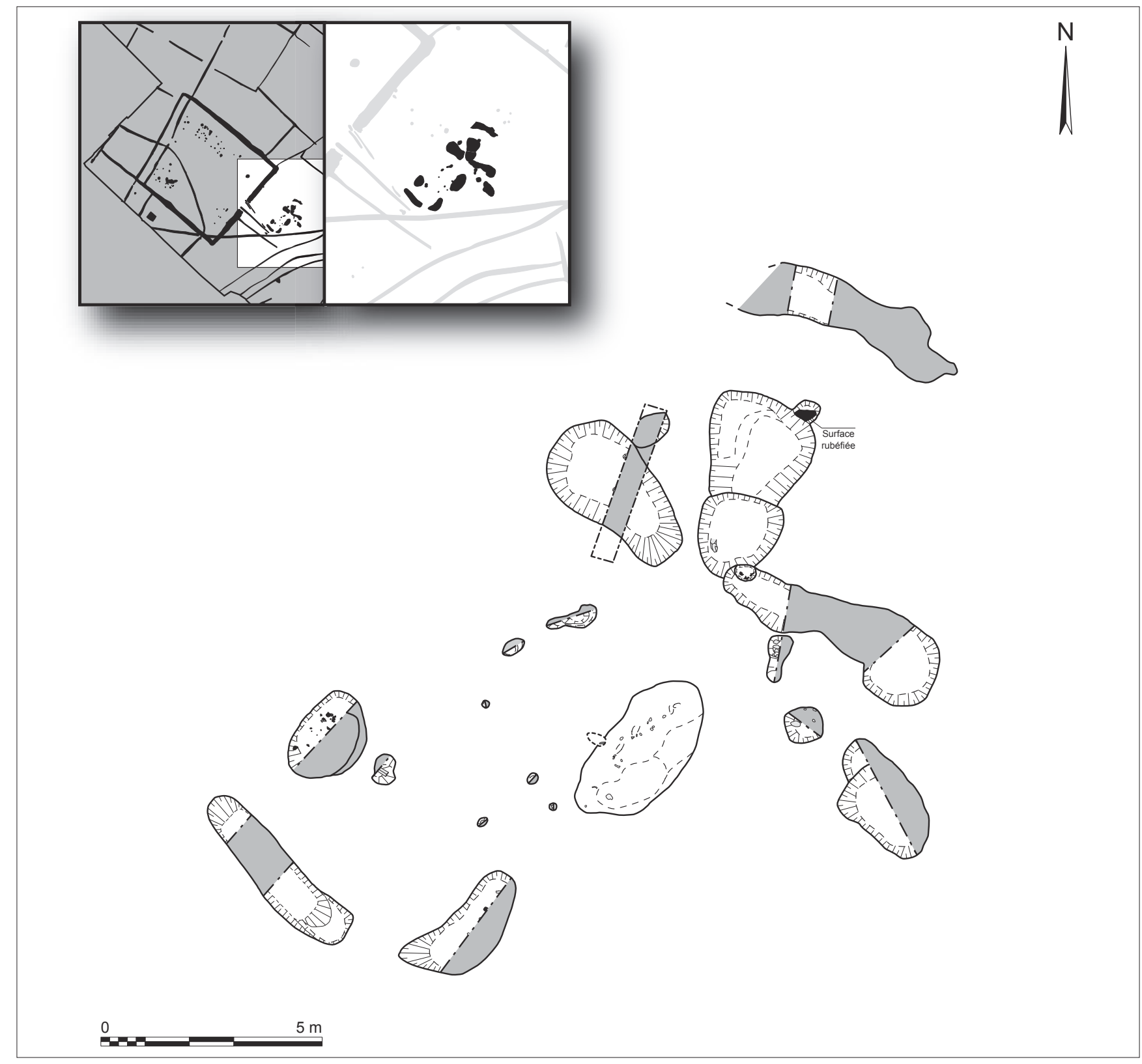

Figure 13 : Ensemble de creusements établis à proximité de l'accès à l'enclos. DAO D. Pfost, Inrap. Figure 13: Excavations located near the entrance to the enclosure.

des tombes "isolées " ou des groupes de sépultures sont signalés, en contexte régional, sur différents habitats ruraux du premier Moyen $\hat{A g e}^{42}$ : il en va ainsi du gisement de "Vauvert " à Château-Gontier ${ }^{43}$ où une probable sépulture

42. Guerin F., "L'organisation spatiale des établissements ruraux du Moyen Âge ", VAlais A. (dir.), L'habitat rural au Moyen Âge dans le NordOuest de la France, tome 1 : Les synthèses, Rennes, PUR, coll. «Archéologie et Culture ", Rennes, 2012, , p. 66-74.

43. Valais A., Arthuis R., Nauleau J.-F., Morera-Vinçotte I., « Les enclos du haut Moyen Âge de Vauvert à Château-Gontier (Mayenne) ", Revue archéologique de l'Ouest, ${ }^{\circ}$ 26, Rennes, 2009, p. 221. rapportée à la phase tardive de l'occupation (VIII siècle), a été aménagée dans le comblement d'un fossé d'enclos; de la même manière, le site des "Landes de Beaulieu ${ }^{44}$ " (Beaulieu-sur-Layon), fréquenté entre la fin $\mathrm{du}^{\mathrm{e}}$ et le début du $\mathrm{Ix}^{\mathrm{e}}$ siècle, a-t-il livré deux tombes aux caractéristiques architecturales très proches de celles issues du "plateau de la Mayenne »; enfin, sur le «village » des " Grandes

44. Guérin F., Beaulieu-sur-Layon (Maine-et-Loire), Les Landes de Beaulieu, DFS de fouille, Afan/SRA des Pays-de-la-Loire, Nantes, 1999, p. $49-52$. 


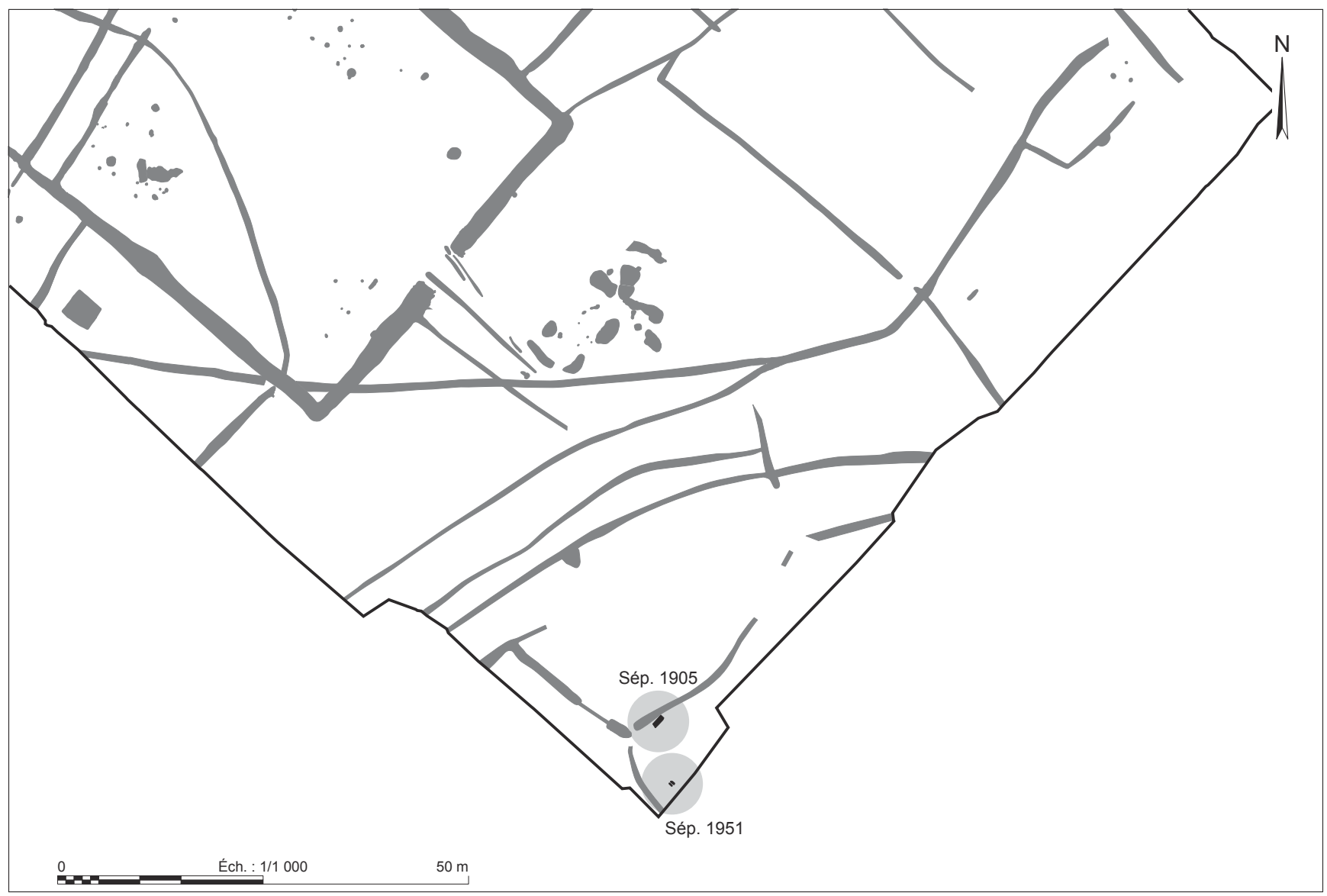

Figure 14 : Localisation des deux sépultures. DAO D. Pfost, Inrap. Figure 14: Location of the two burials.

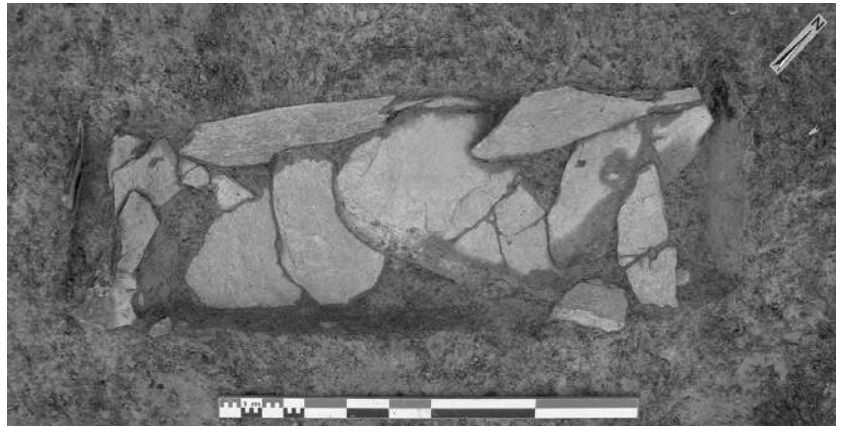

Figure 15 : Vue de l'une des deux sépultures repérées au sud de la fouille. Cliché A. Boterf, Inrap.

Figure 15: View of one of the two burials to the south of the excavation area.

Valinières " (Saint-Sylvain-d'Anjou), occupé entre le viI ${ }^{\mathrm{e}}$ et le $\mathrm{XII}^{\mathrm{e}}$ siècle, certaines des quelque cinquante inhumations recensées, comportaient des coffres ou des traces de coffre en schiste ardoisier ${ }^{45}$.

45. GÉHAn T., «Archéologie et Autoroute A11 », Sites, nº 38, 1987, p. $9-13$.
Certes attesté dès le Bas-Empire ${ }^{46}$, l'usage de l'ardoise dans l'architecture funéraire tend à se développer en Anjou durant le haut Moyen Âge, pour décliner ensuite, et disparaître au cours du Moyen Âge central ${ }^{47}$. Or, cette dernière période - tout comme d'ailleurs la fin de l'Antiquité - est totalement absente du site du « Plateau de la Mayenne ». En vertu de ce constat, il apparait donc vraisemblable que les deux structures en schiste ardoisier détectées sur le gisement se rapportent bien à sa période d'occupation principale, à savoir un intervalle chronologique débutant entre la fin du $\mathrm{VII}^{\mathrm{e}}$ et se terminant au cours du $\mathrm{IX}^{\mathrm{e}}$ siècle.

\section{Un réseau parcellaire (fig. 16)}

Occupant la majeure partie de l'espace décapé, un réseau parcellaire orthogonal plus ou moins régulier se développe autour de l'habitat. Si, au contact de ce dernier, le réseau fossoyé montre une dynamique parfaitement congruente à celle

46. Prigent D. et Hunault J.-Y., La mort : voyage au pays des vivants, pratiques funéraires en Anjou, Angers, 1996, p. 28.

47. Ibid., p. 34. 
Figure 16 : L'enclos et le parcellaire environnant (VII ${ }^{\mathrm{e}}-\mathrm{IX}^{\mathrm{e}}$ siècle).

DAO D. Pfost, Inrap. Figure 16: The enclosure and field system $\left(7^{\text {th }}-9^{\text {th }}\right.$ century).

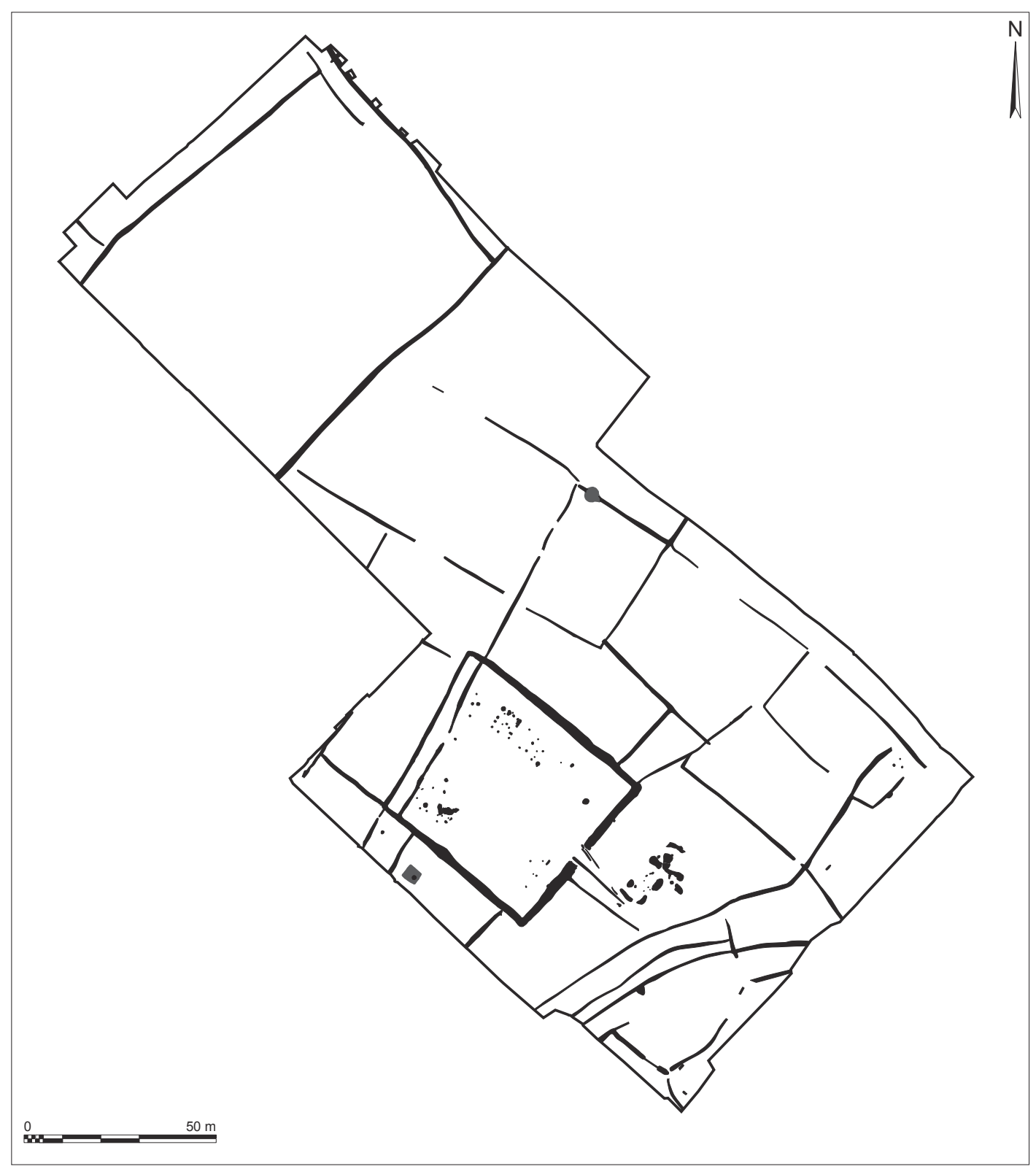

de l'enclos, aux confins nord-ouest et sud-est de la fouille, les fossés tendent à s'en écarter quelque peu.

Quoi qu'il en soit, sur le pourtour immédiat de l'enclos, les fossés, régis par des orientations à dominante NO/SE d'une part, et NE/SO, de l'autre, offrent des largeurs oscillant entre 0,45 mètre et 1,18 mètre, pour des profondeurs s'échelonnant de 0,08 mètre à 0,68 mètre. Pourvus de profils associant fond plat ou légèrement incurvé et parois obliques ou évasées, les creusements délimitent des parcelles rectangulaires ou trapézoïdales, qui - pour celles dont la surface a pu être appréhendée - s'inscrivent entre 160 et 1408 mètres carrés. En dehors des abords sud-est et sud-ouest de l'enclos, les autres périmètres ne comportent que peu ou pas de vestiges.
Dépourvus de contact avec les tronçons précédemment évoqués, les fossés repérés sur la frange sud-est du décapage, montrent des orientations légèrement divergentes : ainsi, se développent-ils selon l'axe ONO/ESE, d'une part, et selon l'orientation NNE/SSO, d'autre part. Dotés de profils assez semblables aux précédents, ils montrent des largeurs comprises entre 0,40 mètre et 1,50 mètre, pour des profondeurs qui s'étagent de 0,07 mètre à 0,66 mètre. Tendant à suivre un tracé légèrement curviligne, deux fossés strictement parallèles et distants de 2,50 mètres à 3 mètres, semblent délimiter un espace de circulation; dans cette hypothèse, ce chemin présumé pourrait avoir été aussi bien dédié à la desserte des parcelles environnantes que destiné à faciliter l'accès à l'enclos d'habitat. 
Établis aux confins nord-ouest de la fouille, différents fossés régis par des orientations proches de celles du dispositif central, n'en offrent pas moins une légère discordance par rapport aux tronçons parcellaires implantés un peu plus au sud. S'étirant du nord-est au sud-ouest, et du nord-ouest au sud-est, ces creusements affichent des largeurs comprises entre 0,70 mètre et 1,75 mètre, pour des profondeurs qui s'échelonnent de 0,30 mètre à 0,65 mètre. Par comparaison avec le reste du canevas fossoyé, le maillage parcellaire septentrional apparaît beaucoup plus lâche : ainsi, l'écart entre deux axes strictement parallèles, affecte-t-il une distance minimum de 66 mètres. Dans ces espaces ainsi matérialisés, aucune structure du premier Moyen Âge ne vient prendre place; il en ressort donc l'image d'un périmètre faiblement occupé, à l'instar d'ailleurs d'une bonne partie des abords immédiats de l'habitat (fig. 16).

\section{APPROCHE SYNTHÉTIQUE DU SITE}

\section{Les espaces domestiques et/ou artisanaux}

Révélés principalement par la présence de rejets, les espaces domestiques et/ou artisanaux se concentrent au cœur de l'enclos quadrangulaire ainsi que sur ces marges méridionales.

Véritable " cœur " domestique du gisement, l'enceinte regroupe le seul bâtiment avéré du site ainsi que divers creusements tels que fosses et trous de poteau; en dehors de ces éléments, une structure de combustion a été également décelée. A contrario, des zones vierges de tout vestige émergent distinctement : ainsi, au centre même de la surface enclose, un espace d'environ 560 mètres carrés établi dans l'axe de l'entrée, fait-il songer à une cour; dans un autre registre, le bord interne du fossé d'enclos montre une «bande " de trois à cinq mètres de large exempte de vestiges, susceptible d'être interprétée comme l'emplacement d'un talus et/ou d'une haie (fig. 16).

Compte tenu du fait que l'édifice et la majeure partie des trous de poteau détectés dans l'enclos prennent place sur la frange nord-est du dispositif, il est possible que ce dernier périmètre ait été orienté avant tout vers des pratiques domestiques; toutefois, faute de témoignages significatifs, cela reste hypothétique.

Dans la moitié sud-ouest de l'enclos, ce sont deux autres zones "spécifiques" qui ont pu être décelées (fig. 16). La plus septentrionale des deux rassemble quelques trous de poteau, des creusements évoquant des structures d'extraction ainsi qu'une fosse riche en rejets charbonneux; également bordé par un fossé bien pourvu en particules de charbon de bois et en scories de fer, ce secteur évoque un espace à vocation plutôt " artisanale ». Pareil constat pourrait être également de mise s'agissant du périmètre méridional, lequel regroupe en effet quelques poteaux et une structure de combustion; là encore, le fossé le plus proche a livré scories et charbons de bois, éléments indicatifs d'une petite activité métallurgique peut-être liée à la réparation des outils.

Ainsi, l'enclos d'habitat réunit-il - autour d'une probable cour - une zone de bâti d'un côté et diverses structures artisanales de l'autre; reste que l'enclos ne regroupe pas la totalité des vestiges révélant des pratiques domestiques ou artisanales. En effet, bien que situés à proximité immédiate de l'habitat, certains éléments particuliers s'inscrivent à l'extérieur de celui-ci. Suggérant l'existence d'espaces annexes en lien plus ou moins étroit avec la sphère domestique, ils apparaissent au sud-ouest sous la forme d'une structure de combustion et d'un puits, tandis qu'au sud-est, ils se matérialisent au travers diverses fosses et quelques trous de poteau.

Implanté à proximité de l'enclos, le puits vient renforcer l'image domestique du périmètre (fig. 17). Parfois considéré comme une structure d'équipement communautaire ${ }^{48}$, le puits semble, dans le cas du site du «Plateau de la Mayenne ", plutôt correspondre à un dispositif à usage privatif. Établi dans une parcelle contigüe à l'enclos d'habitat, le puits s'inscrit non loin d'une structure de combustion.

Second secteur «spécifique " probablement lié à l'enclos, l'espace localisé au sud-est, auprès de l'accès à l'habitat, regroupe en effet un ensemble de creusements comportant rejets domestiques et traces évoquant des pratiques artisanales (fig. 16). Parmi elles, il a été noté la présence de scories en calotte - indicatives d'une activité de forge - et d'un fragment de polissoir en verre, caractéristique du travail des étoffes.

Bien que situés en position secondaire, ces différents témoignages attestent l'existence de travaux artisanaux en liaison avec la vie quotidienne des habitants. Reste que la fonction primaire des fosses implantées au sud-est de l'enclos demeure énigmatique; bien qu'il ne soit pas impossible que certains de ces creusements relèvent de structures de prélèvements de matériaux (argile, plaquettes de schiste), il est également envisageable que tout ou partie d'entre eux correspondent à des silos : dans cette perspective, le secteur pourrait avoir été notamment dédié au stockage des céréales (fig. 17).

48. Peytremann E., op. cit., vol. 1, p. 295. 
Figure 17 : (Voir planche couleur XIII) Plan interprétatif du site $\left(\mathrm{VII}^{\mathrm{e}}-\mathrm{IX}^{\mathrm{e}}\right.$ siècle $)$. DAO D. Pfost, Inrap.

Figure 17: (See colour plate XIII) Interpretation of the site's features.

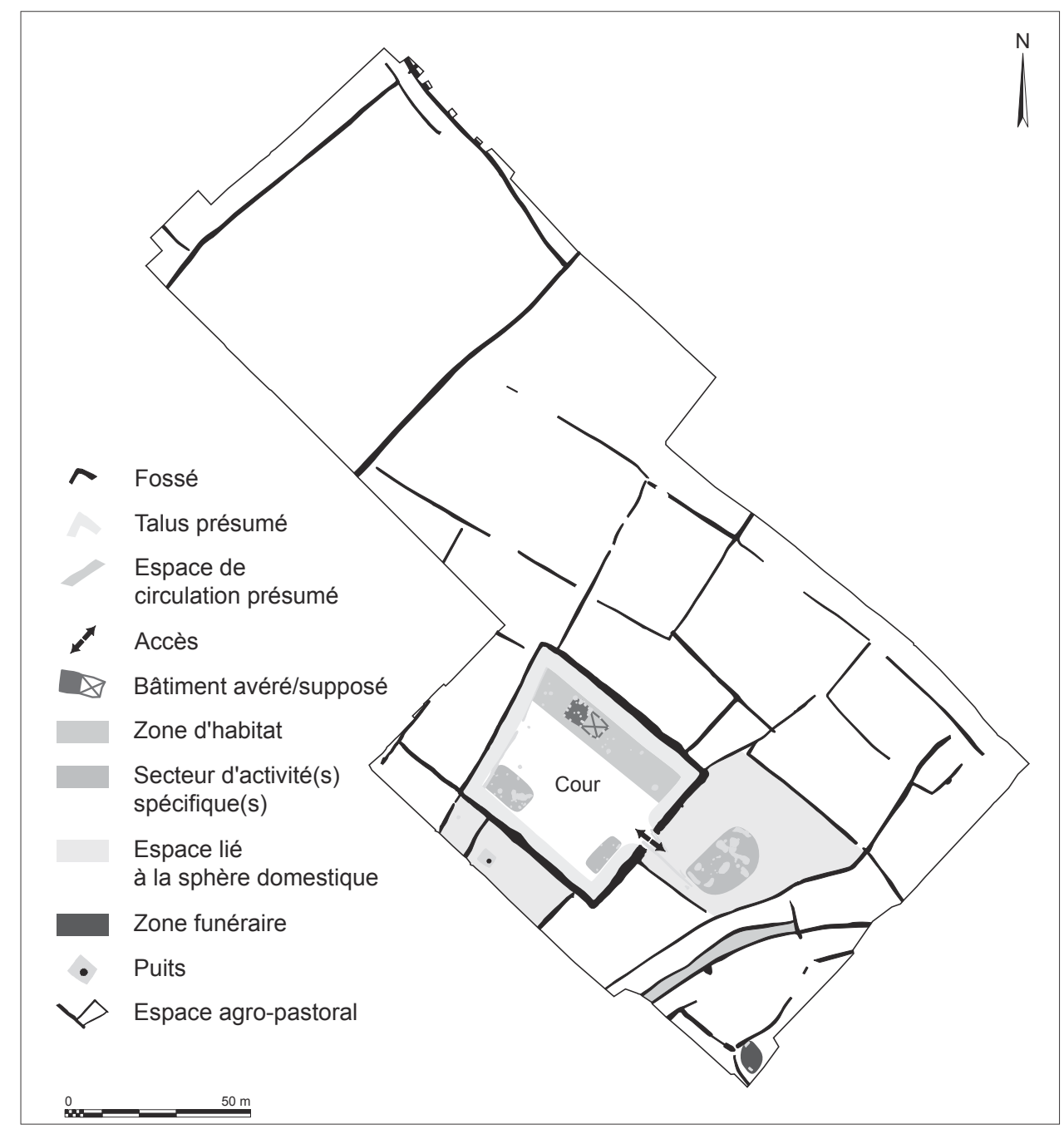

\section{Le maillage parcellaire}

Élément central de l'installation, l'enclos trapézoïdal côtoie, dans ses abords immédiats, un maillage parcellaire dont la trame offre un aspect orthogonal. Pour autant, cette dynamique n'exclut ni une certaine diversité morphologique des parcelles - le plus souvent rectangulaires, parfois trapézoïdales - ni surtout une grande variabilité des superficies encloses, lesquelles s'étagent en effet entre 160 et 1408 mètres carrés avec - comme valeurs les plus « fréquentes »- des surfaces comprises entre 814 et 927 mètres carrés. Ces dernières, comme d'ailleurs la plus importante mentionnée plus haut, se rapprochent des superficies couvertes par les enclos de "Vauvert " à Château-Gontier en Mayenne, où elles occupent entre 850 et 1500 mètres carrés ${ }^{49}$, soit des valeurs proches de

49. Valais A., Arthuis R., Nauleau J.-F. et Morera-Vinçotte I., "Les enclos du haut Moyen Âge de Vauvert à Château-Gontier (Mayenne) ", Revue archéologique de l’Ouest, n²6, Rennes, 2009, p. 226. l'arpent, mesure variant, selon les régions, entre 900 et 1400 mètres carrés ${ }^{50}$.

Hormis les parcelles sises auprès des côtés sud-ouest et sud-est de l'enclos, toutes les autres ne recelaient que peu ou pas de structures du haut Moyen Âge; en outre, la plupart des fossés parcellaires du secteur central n'ont fourni qu'un mobilier rare et fragmentaire suggérant une orientation avant tout agro-pastorale des surfaces encloses. Faute de données complémentaires, il s'avère difficile de préciser la nature des activités effectuées au sein de ces espaces; reste qu'en vertu du contexte du site, on peut supposer - sans trop de risque de se tromper - qu'ils pouvaient aussi bien accueillir la pratique de la culture (champ, verger, jardin) que celle de l'élevage.

Loin d'être spécifique au site du " Plateau de la Mayenne ", cette disposition orthogonale du parcellaire se retrouve en effet sur d'autres sites du haut Moyen Âge, qu'il s'agisse de

50. Ibid., p. 226. 
gisements voisins comme ceux des "Grandes Valinières ${ }^{51}$ " à Saint-Sylvain-d'Anjou et des "Landes de Beaulieu ${ }^{52}$ " à Beaulieu-sur-Layon dans le Maine-et-Loire, ou d'établissements un peu plus éloignés, à l'instar de "Vauvert ${ }^{53}$ " à Château-Gontier (Mayenne), de "La Cocherais ${ }^{54}$ " à Tinténiac (Ille-et-Vilaine) ou encore du "Teilleul ${ }^{55}$ " à Montours (Ille-et-Vilaine). Pour autant, aucun d'eux n'offre une telle organisation "radio-quadrillée $e^{56}$ ", organisation hiérarchisée et centrée autour d'un enclos "principal ».

S'écartant quelque peu de la dynamique prévalant à proximité de l'habitat, les fossés établis aux marges nord-ouest de la fouille, cernent des espaces de dimensions nettement plus importantes, puisque, bien qu'incomplète, la parcelle la mieux perçue se développe ainsi sur une largeur variant de 66 à 70 mètres, pour une longueur supérieure à 81 mètres, soit une superficie dépassant les 5500 mètres carrés. Il en va de même des parcelles voisines de la précédente, lesquelles, bien que plus mal appréhendées encore, semblent néanmoins offrir des dimensions tout aussi importantes, sachant qu'elles oscillent entre 70 et 72 mètres de large, soit des valeurs là encore nettement plus élevées que celles notées autour de l'enclos d'habitat.

Ne renfermant apparemment aucune structure du haut Moyen Âge, les parcelles septentrionales semblent avoir été vouées à des pratiques agro-pastorales; en vertu de leur module " conséquent ", il n'est pas interdit de penser qu'elles ont pu accueillir cultures et zones de pacage, moyennant, le cas échéant, l'installation de clôtures temporaires, à l'image des dispositifs attestés plus tardivement par l'iconographie du second Moyen Âge ${ }^{57}$.

Situés à l'opposé des précédents, les fossés de la frange méridionale de la fouille montrent une dynamique légèrement curviligne probablement induite par la présence

51. Brodeur J. et Thooris C., " Habitats du haut Moyen Âge en Paysde-la-Loire : état de la recherche ", in Lorren C., Perin P. (dir.), L'habitat rural du haut Moyen Âge (France, Pays-Bas, Danemark et Grande-Bretagne), Actes des XIVé Journées internationales d'Archéologie mérovingienne, Guiryen-Vexin et Paris, 4-8 février 1993, tome IV des Mémoires publiés par l'AFAM, Paris, 1995, p. 167, 168.

52. Guérin F., Beaulieu-sur-Layon (Maine-et-Loire), Les Landes de Beaulieu, DFS de fouille, Afan/SRA des Pays-de-la-Loire, Nantes, 1999, p. 29-43.

53. Valais A., Arthuis R., Nauleau J.-F. et Morera-Vinçotte I., « Les enclos du haut Moyen Âge de Vauvert à Château-Gontier (Mayenne) ", Revue archéologique de l'Ouest, n² 26, Rennes, 2009, p. 208-215.

54. Le Boulanger F. et Provost A., "Un village carolingien à la Cocherais en Tinténiac ", Dossier du CeRAA, n 20, 1992, p. 91-93.

55. Cattedu I. (dir.), op. cit., p. 36-39.

56. Watteaux M., La dynamique de la planimétrie parcellaire et des réseaux routiers en Vendée méridionale. Études historiographiques et recherches archéo-géographiques, Mémoire de thèse, sous la direction de G. Chouquer, université de Paris 1 - Panthéon-Sorbonne, Paris, 2009, vol. 1, p. 510514.

57. Mane P., Le travail à la campagne au Moyen Âge, étude iconographique, Paris, 2006, p. 95. d'un chemin de 2,50 mètres à 3 mètres de large matérialisé par deux fossés. Au voisinage de ces derniers, le maillage parcellaire, plus irrégulier, ne recèle là encore qu'un petit nombre de structures; parmi elles, on signalera la présence, à l'extrémité sud du décapage, de deux sépultures ainsi que de quelques petits ensembles de creusements aux fonctions mal définies.

\section{Orientations fonctionnelles}

Au regard des données de la fouille et des résultats du diagnostic ${ }^{58}$, l'implantation du " Plateau de la Mayenne " apparaît comme un habitat agricole isolé. Relativement bien perçue, la fonction domestique transparaît notamment par le biais d'un puits, sis à proximité immédiate de l'enclos, mais aussi et surtout au travers la présence de nombreux rejets de céramiques (fig. 18), détectés principalement dans les fossés de l'enclos et dans les différents creusements établis non loin de l'accès à l'habitat.

À l'inverse, les activités agricoles se manifestent de façon beaucoup plus ténue; de fait, en l'absence d'outil agricole, les travaux des champs ne sont illustrés que de manière indirecte, en l'occurrence sous la forme de quelques fragments de meules, indicatives du traitement des céréales, et sous celle de pierres à aiguiser (fig. 19), destinées à l'affutage des lames, dont celles - probablement - de l'équipement agricole de base (faucille, serpe, entre autres...). À ces quelques artefacts pourraient éventuellement s'ajouter une partie des fosses sises au sud-est de l'enclos, et dont le regroupement semble évoquer une zone d'ensilage; reste qu'une pareille interprétation demeure néanmoins sujette à caution.

Certes présent sur le gisement, "l'artisanat " ne se manifeste cependant qu'au travers de la métallurgie et de l'activité textile. La première, qui apparaît comme étant la plus marquée, s'exprime sous forme de scories en calotte, caractéristiques d'une activité de forge. S'exerçant aussi bien au cœur de l'enclos que dans le périmètre établi non loin de l'entrée, l'activité métallurgique devait être orientée notamment vers la réparation des outils agricoles.

Nettement plus discrète, l'activité textile n'a en effet été perçue que grâce à la présence d'un lissoir en verre découvert lors du diagnostic ${ }^{59}$. Destiné à l'assouplissement des étoffes, cet objet provient de l'une des fosses ménagées au sud-est de l'enceinte. Pour le reste, il n'a été détecté aucune autre trace indicative de la pratique du filage ou du tissage.

À l'instar de différents sites des VII $^{\mathrm{e}}$-VIII ${ }^{\mathrm{e}}$ siècles de la moitié nord de la France ${ }^{60}$, le gisement du «Plateau de la Mayenne » a donc livré quelques indices d'activités artisa-

58. Dubillot X., op. cit.

59. Ibid., p. 42, 43.

60. Peytremann E., op. cit., vol. 1, p. 345, 346. 


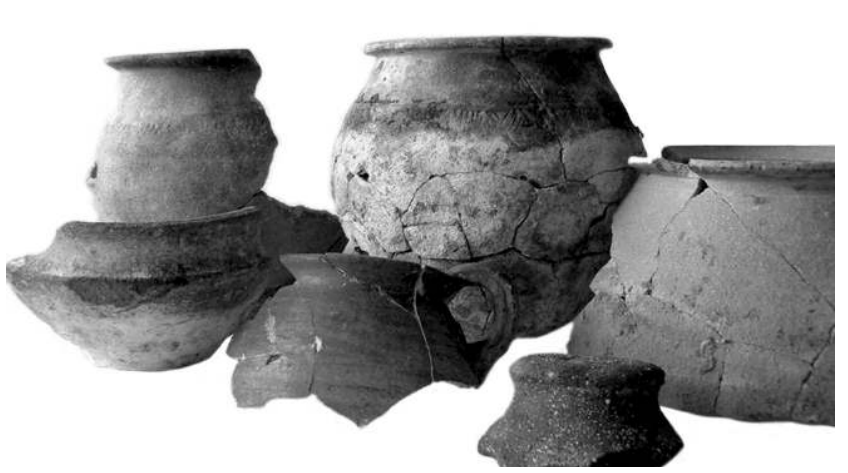

Figure 18 : Vue d'une partie des formes céramiques présentes sur le site. Cliché E. Coffineau, Inrap.

Figure 18: View of the site's pottery.

nales. Celles-ci, s'exerçant au sein de d'habitat, mais également au sud-est de ce dernier, semblent avoir été avant tout destinées à répondre aux besoins des habitants; en cela, l'implantation angevine ne diffère guère de la plupart des installations des $\mathrm{VII}^{\mathrm{e}}$-VIII ${ }^{\mathrm{e}}$ siècles où sont attestées de telles pratiques artisanales.

\section{Une implantation indicative d'une unité agricole}

Apparaissant isolée, l'installation du "Plateau de la Mayenne " dépasse non seulement le cadre arbitraire du décapage, mais aussi les neuf hectares (fig. 20) au sein desquels ont été perçues, lors du diagnostic, les différentes traces du haut Moyen Âge. Par le caractère quelque peu " ostentatoire " de son enclos d'habitat et la qualité de son mobilier céramique ${ }^{61}$, l'implantation pourrait témoigner d'une " aisance relative " de ses habitants. Néanmoins, la rareté des autres restes mobiliers ainsi que la présence d'un seul bâtiment avéré tendent quelque peu à pondérer cette image.

Reste que l'ensemble témoigne sans doute d'une unité d'exploitation agricole, unité d'exploitation où un habitat, établi au sein d'un enclos fossoyé, apparaît comme l'élément central du dispositif. Il est vrai qu’à proximité, les parcelles s'articulent distinctement en fonction de la dynamique spatiale générée par l'enclos. Accueillant sans doute essentiellement des activités agricoles et/ou pastorales (?) le maillage parcellaire, d'abord congruent à l'enclos central, tend progressivement à s'en différencier au fur et à mesure qu'il s'écarte du cœur de l'habitat. Dans un autre registre, il convient de signaler qu'au nord de ce dernier, les parcelles offrent des modules beaucoup plus importants que ceux des autres secteurs, signalant peut-être ainsi l'existence d'espaces voués à différentes productions agro-pastorales (?) ;

61. Coffineau E., op. cit., p. 173.

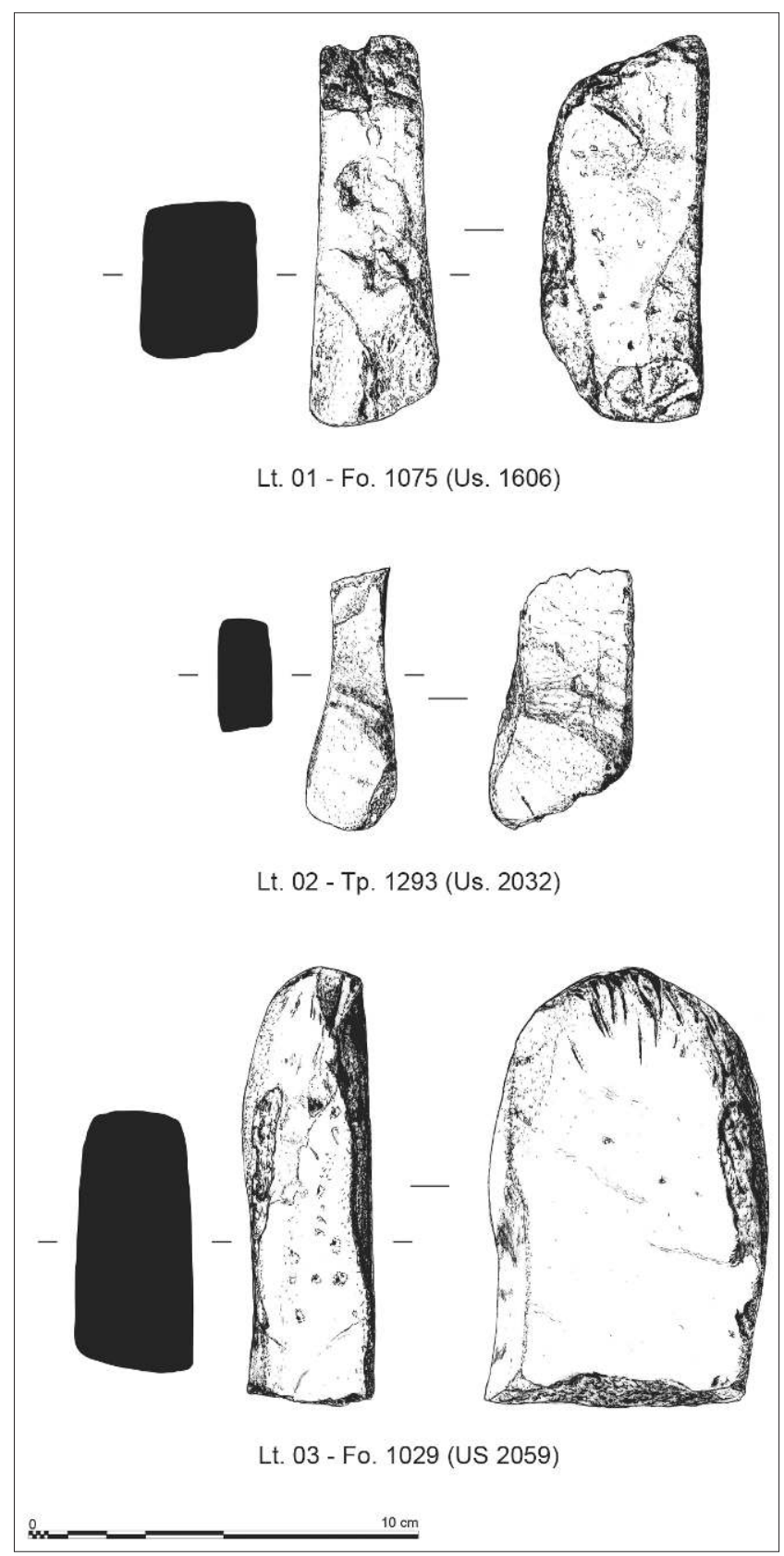

Figure 19 : Trois pierres à aiguiser. Dessins J.-N. Griffish, Inrap. Figure 19: Three sharpening stones.

pour autant, faute de données probantes, un tel postulat demeure à ce jour hautement conjectural.

\section{Conclusion}

Isolé, l'établissement du " Plateau de la Mayenne " s'intègre dans un environnement faiblement marqué par les témoignages gallo-romains; pour le moins ténus, ceux-ci soulignent l'existence d'un hiatus de plusieurs siècles entre 


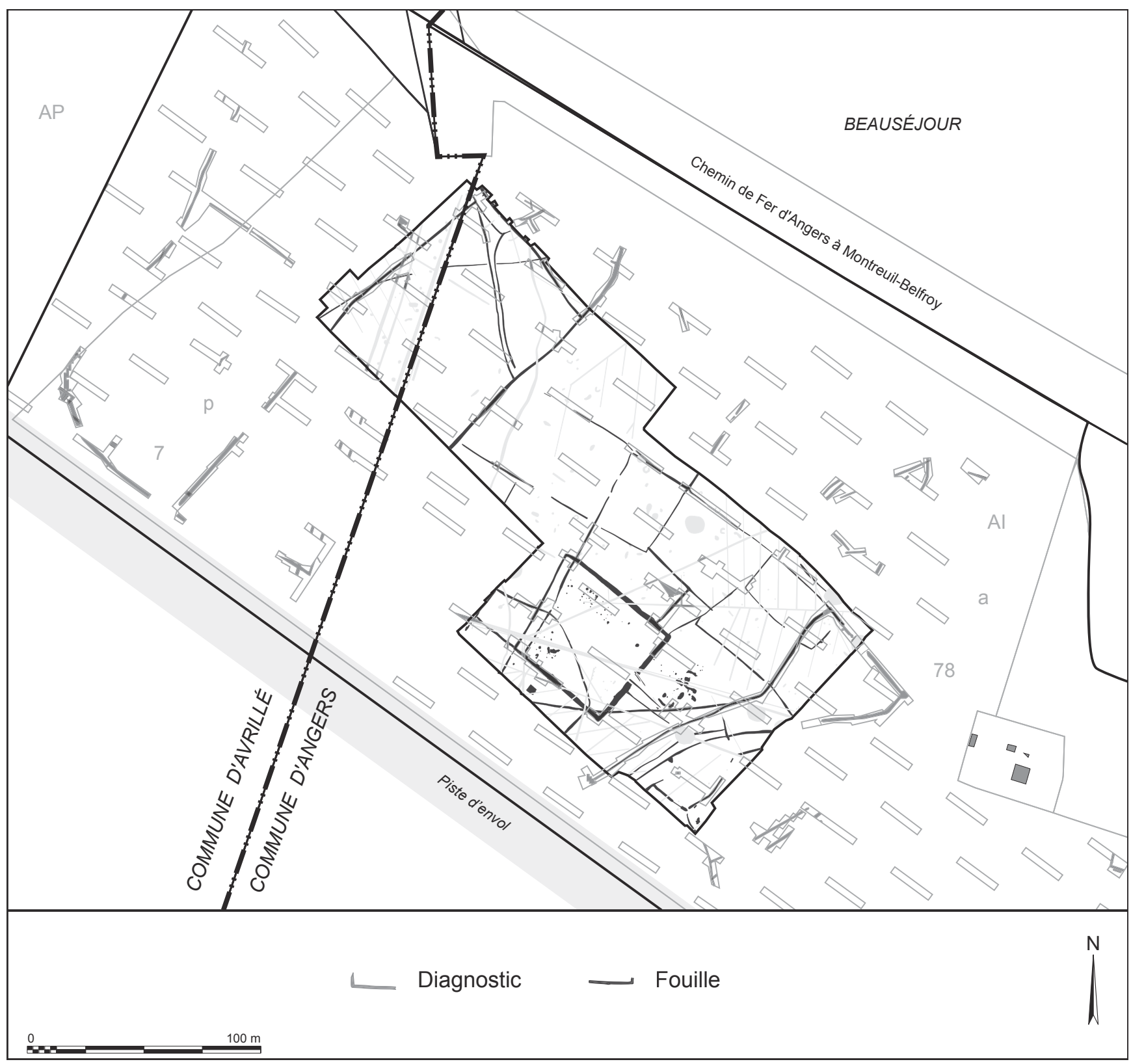

Figure 20 : Plan général des vestiges observés lors du diagnostic et de la fouille. DAO D. Pfost, Inrap. Figure 20: General plan of the features of the evaluation and the excavation.

vestiges antiques et premiers éléments du haut Moyen Âge. Ces derniers, indicatifs des $\mathrm{VI}^{\mathrm{e}}-\mathrm{VII}^{\mathrm{e}}$ siècles, ne semblent pas avoir été particulièrement nombreux, puisqu'ils se limitent à trois fossés curvilignes orientés sans doute vers le drainage et la délimitation d'espaces à vocation prioritairement agropastorale.

En réalité, c'est principalement vers la fin du viI ${ }^{\mathrm{e}}$ siècle, mais aussi et surtout dans le courant du siècle suivant que se met véritablement en place l'installation du « Plateau de la Mayenne ». Celle-ci, structurée autour d'un habitat matéria- lisé par un enclos trapézoïdal assez conséquent, se caractérise par une organisation paysagère à dynamique orthogonale accueillant - probablement - des activités de culture et d'élevage. Reste que ce sont principalement les pratiques domestiques qui ont été perçues, notamment grâce au mobilier céramique; d'autres indices attestent par ailleurs la présence d'une activité de forge ainsi que celle du travail des étoffes.

Quoi qu'il en soit, si l'occupation de l'établissement paraît se renforcer au cours du viII ${ }^{\mathrm{e}}$ siècle, en revanche, le $\mathrm{IX}^{\mathrm{e}}$ marque son déclin et, semble-t-il, son abandon. Sans pou- 
voir en déterminer les causes, il n'est pas impossible que les troubles que connaît alors la région d'Angers au milieu du $\mathrm{IX}^{\mathrm{e}}$ siècle (conflit avec la Bretagne, incursions scandinaves), aient pu exercer une influence sur la disparition de l'installation. Toujours est-il que postérieurement à l'abandon de cette dernière, une zone forestière - dépendant du comte au début du XII ${ }^{e}$ siècle - se met progressivement en place et va occuper les lieux jusqu'à ce qu'intervienne, au début $\mathrm{du} \mathrm{xx}^{\mathrm{e}}$ siècle, l'aménagement de l'ancien terrain d'aviation d'Angers/Avrillé.

Actuellement unique installation agricole du haut Moyen Âge reconnue aux abords immédiats d'Angers, l'implantation du "Plateau de la Mayenne " relève d'une unité d'exploitation dont les limites dépassent les cadres arbitraires de la fouille. De fait, si cette dernière a été l'occasion d'appréhender le cœur du dispositif, elle n'a, en revanche, permis de percevoir qu'une partie des espaces qui lui étaient associés. Ceux-ci, attestés sur plus de neuf hectares, montrent l'importance d'un établissement qu'il s'avère cependant difficile de qualifier. Plutôt constitutif d'une partie d'un domaine que le centre de celui-ci, il représente un jalon appréciable à la compréhension des différentes formes d'habitat rural de l'ouest de la France, à une période située à la charnière des dynasties mérovingienne et carolingienne.

\section{Bibliographie}

Brodeur J. et Thooris C., 1995 - " Habitats du haut Moyen Âge en Pays de la Loire : état de la recherche ", in Lorren C., Perin P. (dir.), L'habitat rural du haut Moyen Âge (France, Pays-Bas, Danemark et Grande-Bretagne), Actes des XIVe Journées internationales d'Archéologie mérovingienne, Guiry-en-Vexin et Paris, 4-8 février 1993, tome IV des Mémoires publiés par l'AFAM, Paris, 1995, p. 167- 174.

Brodeur J., 2011 - «Les abords nord-ouest d'Angers entre la fin de l'âge du Fer et le haut Moyen Âge ", in Guerin F. (dir.), Angers (49007), Avrillé (49015), Maine-et-Loire, ZAC du Plateau de la Mayenne, tranche 1 : un établissement rural du haut Moyen Âge aux portes d'Angers, fouille archéologique, Rapport Final d'Opération, Inrap Grand-Ouest, CessonSévigné, p. 40-42.

Catteddu I. (dir.), 200 I - Les habitats carolingiens de Montours et La Chapelle-Saint-Aubert (Ille-et-Vilaine), DAF n 89, Paris, $235 \mathrm{p}$.

Cavet P., Chauris L., Blaise J., Arnaud A., Gruet M., Lardeux H. et Brosse R., I976 - Carte géologique de la France à 1/50000, feuille d'Angers (454), éditions du BRGM, une carte et une notice de 42 p, Orléans.

Coffineau E., 20 I I - "La céramique du haut Moyen Âge ", in GuÉrin F. (dir.), Angers (49007), Avrillé (49015), Maine-et-
Loire, ZAC du Plateau de la Mayenne, tranche 1 : un établissement rural du haut Moyen Âge aux portes d'Angers, fouille archéologique, Rapport Final d'Opération, INRAP GrandOuest, Cesson-Sévigné, p. 173-183.

Comte F., I 997 - "Le site, la chapelle et l'enclos des lépreux", SaintLazare : Histoire d'une Léproserie et d'un faubourg d'Angers, XIIXVII siècle, ARDA n ${ }^{\circ} 1$, Angers, p. 8-15.

Desmazières O., I 925 - "Inventaire des haches de pierre polie du département de Maine-et-Loire, et relevé des polissoirs ", Bulletin de la Société d'Études Scientifique d'Angers, LV, p. 94.

Deloze V., 20 I I - "Environnement naturel ", in Guerin F. (dir), Angers (49007), Avrillé (49015), Maine-et-Loire, ZAC du Plateau de la Mayenne, tranche 1 : un établissement rural du haut Moyen Âge aux portes d'Angers, fouille archéologique, Rapport Final d'Opération, Inrap Grand-Ouest, CessonSévigné, p. 33-39.

Dubillot X., 2006 - Angers/Avrillé (Maine-et-Loire), "ZAC du Plateau de la Mayenne ", Tranche 1, 49007 et 49015, diagnostic archéologique, Rapport Final d'Opération, Inrap GrandOuest/SRA des Pays de la Loire, Nantes, 54 p.

Fricot L., 2007 - Avrillé, La Meignanne, La Membrolle-surLonguenée, Montreuil-Juigné, Le Plessis-Macé (Maine-et-Loire), $R D 775$ (ex RN 162) - Aménagement en 2 X 2 voies entre Avrillé et La Membrolle-sur-Longuenée, déviation de l'ETAS, Rapport de diagnostic d'archéologie préventive, SAD de Maine-etLoire/SRA des Pays de la Loire, Angers, p. 60-65.

GÉHan T., I 987 - «Archéologie et Autoroute A11 ", Sites, nº 38, p. 9-13.

GuÉRIN F., I 999 - Beaulieu-sur-Layon (Maine-et-Loire), "Les Landes de Beaulieu ", DFS de fouille, AFAN/SRA des Pays de la Loire, Nantes, 112 p.

Guérin F., 2006 - Angers (Maine-et-Loire), ZAC du Plateau des Capucins, tranche 1, Rapport de Diagnostic Archéologique, Inrap Grand-Ouest/SRA des Pays de la Loire, Nantes, p. 4.

Guerin F., 2008 - Angers (Maine-et-Loire), ZAC du Plateau des Capucins, lot $n^{\circ} 2$, rapport de diagnostic archéologique, Inrap Grand-Ouest/SRA des Pays de la Loire, Nantes, 95 p.

Guerin F., 20I I - Angers (49007), Avrillé (49015), Maine-etLoire, ZAC du Plateau de la Mayenne, tranche 1 : un établissement rural du haut Moyen Âge aux portes d'Angers, fouille archéologique, Rapport Final d'Opération, Inrap GrandOuest, Cesson-Sévigné, 338 p.

Guerin F., 20I 2 - «L'organisation spatiale des établissements ruraux du Moyen Âge ", in VAlAis A. (dir.), L'habitat rural au Moyen Âge dans le nord-ouest de la France, tome 1 : Les synthèses, Rennes, Presses Universitaires de Rennes, coll. «Archéologie et Culture », p. 19-84.

Hervé M.-L., 2004 - Angers (Maine-et-Loire), La Gatellière, rapport de fouille archéologique, Inrap Grand-Ouest/SRA des Pays de la Loire, Nantes, 48 p. 
Le Boulanger F. et Provost A., I 992 - «Un village carolingien à la Cocherais en Tinténiac ", Dossier du CeRAA, no 20, p. 87-117.

Letho-Duclos Y., 2003 - Avrillé (Maine-et-Loire), "La Livonnière " rapport de diagnostic archéologique, Inrap GrandOuest/SRA des Pays de la Loire, Nantes, n.p.

Mane P., 2006 - Le travail à la campagne au Moyen Âge, étude iconographique, Paris, $471 \mathrm{p}$.

Pétorin N., Mercier-Doucet F. et Pean E., 2004 - Cholet (Maine-et-Loire), ZAC L'Ecuyère, diagnostic archéologique, Rapport Final d'Opération, Inrap Grand-Ouest/SRA des Pays de la Loire, Nantes, 34 p.

Pétorin N., 20 Io - Angers, Maine-et-Loire, Beauséjour, Etablissement rural de La Tène finale, fouille archéologique, Rapport Final d'Opération, Inrap Grand-Ouest, CessonSévigné, $77 \mathrm{p}$.

Peytremann E., 2003 - Archéologie de l'habitat rural dans le nord de la France du IV au XII siècle, tome XIII des Mémoires publiés par l'Association Française d'Archéologie Mérovingienne, 2 vol., Saint-Germain-en-Laye.

PorT C., I 878 - Dictionnaire historique, géographique et biographique de Maine-et-Loire, Angers, t. I.
Prigent D. et Hunault J.-Y., I 996 - La mort : voyage au pays des vivants. Pratiques funéraires en Anjou, Angers, 151 p.

Tonnerre N.-Y., à paraître - "L'Anjou de l'Antiquité tardive au très haut Moyen Âge ", Actes des XXII ${ }^{e}$ journées internationales d'Archéologie Mérovingiennes tenues à Angers en 2001.

Tourneur J., 2007 - Angers/Avrillé (Maine-et-Loire), "ZAC du Plateau de la Mayenne ", Tranche 3, rapport de diagnostic archéologique, Inrap Grand-Ouest/SRA des Pays de la Loire, Nantes, 33 p.

Valais A., I997 - Distré (Maine-et-Loire), "Les Murailles ", DFS de sauvetage urgent, tomes 1 et 2, AFAN/SRA des Pays de la Loire, Nantes.

Valais A., Arthuis R., Nauleau J.-F. et Morera-Vinçotte I., 2009 - « Les enclos du haut Moyen Âge de Vauvert à ChâteauGontier (Mayenne) ", Revue archéologique de l'Ouest, $\mathrm{n}^{\circ}$ 26, Rennes, p. 205-227.

Watteaux M., 2009 - La dynamique de la planimétrie parcellaire et des réseaux routiers en Vendée méridionale. Etudes historiographiques et recherches archéogéographiques, Mémoire de thèse, sous la direction de G. Chouquer, Université de Paris 1 Panthéon-Sorbonne, Paris, vol. 1, 569 p.

Zusammenfassung: Der Fundplatz „Plateau de la Mayenne”, eine Siedlung des 7.-9. Jh. n. Chr. in der Nachbarschaft von Angers (Maineet-Loire, France) - Die Fundstelle des "Plateau de la Mayenne", die im Nordwesten von Angers (Maine-et-Loire, Frankreich) liegt, wurde im Jahr 2006 von X. Dubillot während einer archäologischen Baggerprospektion entdeckt. Die ländliche Siedlung, die sich mindestens über neun Hektar Grundfläche erstreckt, war 2009 das Ziel einer archäologischen Ausgrabung, die etwa drei Hektar Fläche umfasste. In diesem Bereich konnten das durch eine befestigte Einhegung gekennzeichnete Siedlungszentrum sowie seine unmittelbare Nachbarschaft untersucht werden. Letztere zeichnet sich durch eine orthogonale Flureinteilung aus. Die eigentlichen Siedlungsbefunde waren selten und überwiegend schlecht erhalten. Sie beschränken sich auf einen wohl in HolzLehmbauweise errichteten Pfostenbau, einen Brunnen und möglicherweise eine Ofenanlage. Am Rand der Siedlung wurden einige Gruben sowie zwei Gräber nachgewiesen, die ihr zuzuweisen sind. Die Siedlung wird schließlich vor dem 10. Jh. n. Chr. aufgegeben.

Resumen: El sitio del "Plateau de la Mayenne", un habitat rural de los siglos VII-LX en los afueras de Angers (Maine-et-Loire) - Situado al noroeste de Angers, el sitio "Plateau de la Mayenne" fue descubierto en 2006 por X. Dubillot durante una evaluación arqueológica. Reconocido sobre más de nueve hectáreas, la propiedad rural, fechadas de los siglos VII-IX, fue objeto de una excavación en 2009. Las buisquedas se extendieron en casi tres hectáreas. En este ámbito, ha sido posible estudiar el corazón de la ocupación, materializado por un recinto, y su entorno inmediato, caracterizado por un sistema fragmentado ortogonal. Más bien raras y mal conservadas, las estructuras de hábitat se limitan a un edificio con postes, un pozo y, probablemente, un horno. Reconocidas en los márgenes del hábitat, algunas fosas y dos tumbas también se refieren a la instalación. Ésta fue abandonada antes del siglo X.

Schlüsselwörter: Frühmittelalter, ländliche Siedlung, Einhegung, Gräben, Pfostenbau, Brunnen, Gräber, Fundmaterial.

Palabras clave: Edad Media, vivienda rural, recinto, zanjas, construcción sobre postes, pozos, tumbas, material. 